\title{
„A Place where the Light is constant." Faktoren der Migration von Nigeria nach Europa und Integration durch den Verkauf von Straßenzeitungen am Beispiel von Tirol
}

\author{
Tobias Leo \\ Kerngebiet: Zeitgeschichte \\ eingereicht bei: assoz. Prof. Mag. Dr. Eva Pfanzelter (MA) \\ eingereicht im: SoSe 2017 \\ Rubrik: SE-Arbeit
}

\begin{abstract}
"A Place where the Light is constant." Factors of Migration from Nigeria to Europe and Integration through the Sale of Street Newspapers using the Example of Tyrol

The first part of this work is about the reasons why people want to leave their native Nigeria and start a new life in Europe. Many of today's problems in Nigeria are a product of historical developments and can be explained in a problem-oriented perspective, starting with the beginning of the $19^{\text {th }}$ century. The second part is about the Nigerian migrants who have managed to reach Europe, in this case Austria. It is assumed that we live in a post-migrant society and that for migrants, in the style of Homi Bhabha, third spaces can be opened up. Especially for asylum seekers, there are few opportunities to work. One of these is the sale of street papers, which may be one of those third spaces in which integration can be facilitated. Therefore, five Nigerians who sell the Tyrolean street paper "20er" were interviewed.
\end{abstract}

\section{Einleitung}

Migration und Integration sind seit Jahren heiß diskutierte Themen in Österreich, insbesondere seit dem Sommer 2015, in dem überdurchschnittlich viele Migrant_innen aus Drittstaaten nach Europa zuwanderten. Seit vielen Jahren kommen Menschen aus Afrika aus den verschiedensten Gründen nach Europa und damit auch nach Österreich. Im Zentrum dieser Arbeit steht die Migration von Nigeria nach Tirol. Zunächst 
wird Nigeria selbst in den Mittelpunkt gerückt: Die dortigen Probleme sind zahlreich und tief in der Vergangenheit verwurzelt. Nigeria ist ein ressourcenreiches Land mit großem Potential, dennoch lebt ein beträchtlicher Teil der Bevölkerung in Armut. Es ist bisher nicht gelungen, diesen Reichtum gerecht zu verteilen. Es lohnt sich deswegen, die historische Entwicklung Nigerias ab dem Beginn des 19. Jahrhunderts aus einem problemorientierten Blickwinkel zu betrachten, da sich heutige gesellschaftliche Problemlagen und Konflikte bereits in vorkolonialer Zeit gebildet haben. Ein Ziel dieser Arbeit ist es daher, abseits von einfachen Erklärungen tiefer- und weiter zurückgreifende Faktoren an die Oberfläche zu bringen, um zu verstehen, warum Menschen in ihrem Heimatland keine Zukunft mehr sehen und nach Europa und damit auch nach Österreich auswandern.

Es wird angenommen, dass Österreich eine postmigrantische Gesellschaft ist, d. h. die Gesellschaft definiert sich sowohl positiv als auch negativ v. a. durch Migration und Migrationserfahrungen. Innerhalb dieser von dynamischen Diskursen geprägten Gesellschaft stehen Zuwander_innen im Mittelpunkt und diese werden sowohl gefördert als auch gefordert. Von der österreichischen Regierung wurden zu Beginn des Jahres 2016 verpflichtende Orientierungs- und Wertekurse für anerkannte Asylwerber_innen eingeführt, in denen in acht Stunden - sozusagen im Schnelldurchlauf - „Österreichische Werte" vermittelt werden sollen. Eine erste Orientierung in der Gesellschaft ist ohne Zweifel positiv zu bewerten. Fraglich ist allerdings, ob wenige Stunden dafür ausreichend sind, zumal anerkannte Asylwerber_innen sowie Asylberechtigte vor viel größeren Hürden stehen, als verpflichtende Wertekurse zu besuchen. Die vielzitierte "Leistungsgesellschaft" erschwert es Migrant_innen, insbesondere Asylwerber_innen, durch nachteilige Rahmenbedingungen selbst Leistung zu bringen bzw. sich durch den Zugang zum Arbeitsmarkt in der Gesellschaft zu etablieren und damit in der Gesellschaft Fuß zu fassen. Für Asylwerber_innen ist der Arbeitsmarkt bis auf wenige Ausnahmen verschlossen.

Genau an diesem Punkt setzt diese Arbeit an: Eine der Ausnahmen ist der Verkauf von Straßenzeitungen als selbstständige Tätigkeit. Im Zentrum steht hier die Tiroler Straßenzeitung 20er. Verkauften die ersten Jahre fast ausschließlich bedürftige Österreicher_innen, so nahmen - wie später noch ausgeführt werden wird - ab etwa Mitte der 2000er-Jahre hauptsächlich Migrant_innen diese Gelegenheit vermehrt wahr und damit veränderte sich die Zusammensetzung des Kolporteur_innenpools nachhaltig. Diese Erwerbstätigkeit gibt seither insbesondere anerkannten Asylwerber_innen die Möglichkeit, mittel- und langfristig gestützt auf einem größeren finanziellen Spielraum unabhängiger von den eher marginalen staatlichen Leistungen zu sein, wodurch sie sich auch abseits dieser Arbeit mehr am gesellschaftlichen Leben beteiligen können. Es wird angenommen, dass Integration nicht - wie oft gefordert - von heute auf morgen funktioniert, sondern dass die Komplexität der Gesellschaft dafür Zeit und vor allem Partizipation und Austausch mit der einheimischen Bevölkerung erfordert. Der Verkauf von Zeitungen wird in Anlehnung an Homi Bhabhas Konzept der Dritten Räume in einer postmigrantischen Gesellschaft betrachtet. Diese Dritten Räume öffnen sich in 
den Verkaufs- bzw. Gesprächssituationen und bieten etwa die Möglichkeit zum beiderseitigen kulturellen Austausch oder die Möglichkeit der Neubewertung von kulturellen Autoritätssymbolen wie Stereotypen oder Ressentiments. Ein wichtiger Teil dieser Arbeit sind qualitative Interviews mit fünf nigerianischen 20er-Verkäufern. Die Erkenntnisse dieser Gespräche werden an verschiedenen Stellen immer wieder eingestreut werden. Das Ziel dieser Arbeit ist, zunächst zu verstehen, aus welchen Gründen Menschen Nigeria verlassen und mit welchem Mindset sie Europa erreichen. Es soll dabei aufgezeigt werden, dass die aktuellen Probleme des Landes auch historisch bedingt sind und weit zurückreichen. Angekommen in Österreich wird von ihnen erwartet, sich in einer vielschichtigen und postmigrantischen Gesellschaft so schnell wie möglich zu integrieren. Gleichzeitig finden sie sich meist in prekären Lebenslagen wieder und haben geringe Chancen auf Asyl. Tatsächlich benötigt es für Integration Zeit und Räume in Bhabhas Sinn, die staatlichen Maßnahmen scheinen in dieser Hinsicht aber zu kurz zu greifen. Mithilfe des 20ers, der sich als Hilfe für Menschen in sozialen Notlagen und nicht explizit als Integrationsinstitution begreift, eröffnen sich dennoch Möglichkeiten für Asylwerber_innen, sich aktiv an der Gesellschaft zu beteiligen und zu integrieren.

\section{$1.1 \quad$ Literaturüberblick}

Einen bis in präkoloniale Zeiten zurückreichenden Überblick über die Geschichte Nigerias liefern Toyin Falola und Matthew M. Heaton mit "History of Nigeria"1. Umfassend und detailreich gehen sie dabei auf Themen wie Kolonialismus, Politik, Religion, Wirtschaft, aber auch auf Kunst und Kultur ein. Eine ebenso detaillierte und informative Darstellung ist Eghosa Osaghaes Werk "Crippled Giant"2, in dem er sich mit der politischen Geschichte Nigerias seit 1960 auf lokaler, regionaler und globaler Ebene befasst. In aufschlussreicher Weise arbeitet er unter anderem die kolonialen Kontinuitäten nach der Unabhängigkeit heraus. Stefan Salomon widmet sich in „The Tragedy of Nigerian Society"3 den gesellschaftlichen Problemen Nigerias und erörtert, was für eine Rolle Religion dabei spielt. Über die ökonomische Entwicklung Nigerias gibt der Wirtschaftswissenschaftler Robert Kappel in „Nigeria - Ressourcenreichtum und Wachstum ohne Entwicklung"4 einen informativen Überblick. Julia Staller, die ehemalige Chefredakteurin des 20ers, schreibt in ihrer Diplomarbeit „Erwerbsarbeit als Integrationsinstanz bei Asylwerberlnnen in Österreich"5 über die Schwierigkeiten und Möglichkeiten von Asylwerber_innen bezüglich des Arbeitsmarktes in Verbindung mit dem Integrationsbegriff. Des Weiteren geht sie auch auf die psychosozialen Funktionen von Arbeit ein. Was eine postmigrantische Gesellschaft ist, darüber klärt Naika Foroutan in ihrem Auf-

\footnotetext{
Toyin Falola/Matthew M. Heaton, A History of Nigeria, Cambridge-New York-Melbourne 2008. Eghosa Osaghae, Crippled Giant. Nigeria since Independence, London 1998. Stefan Salomon, The Tragedy of Nigerian Society, in: Andreas Exenberger (Hrsg.), Afrika - Kontinent der Extreme (Edition Weltordnung - Religion - Gewalt 9), Innsbruck 2001, S. 147-168.

4 Robert Kappel, Nigeria - Ressourcenreichtum und Wachstum ohne Entwicklung, in: Matthias Basedas/Robert Kappel (Hrsg), Machtquelle Erdöl. Die Außen-, Innen- und Wirtschaftspolitik von Erdölstaaten (Entwicklungstheorie und Entwicklungspolitik 11), Baden-Baden 2001, S. 141-166.

5 Julia Staller, Erwerbsarbeit als Integrationsinstanz bei Asylwerberlnnen in Österreich am Beispiel der Tiroler Straßenzeitung 20er, Dipl. Innsbruck 2012.
} 
satz „Postmigrantische Gesellschaften"6 auf. Zu guter Letzt definiert Homi Bhabha in seinem theoretisch geprägten, komplexen und einflussreichen Werk "The Location of Culture" ${ }^{\prime 7}$, was Dritte Räume sind.

\subsection{Die Interviews}

Es muss an dieser Stelle klargestellt werden, dass der Autor zum Zeitpunkt des Verfassens dieser Arbeit beruflich als Vertriebsleiter beim 20er arbeitete und daher viel mit den Verkäufer_innen zu tun hatte. Die Interviews sind auf der Basis von Vertrauen und gegenseitiger Wertschätzung zustande gekommen. Die Gesprächspartner erklärten, dass das für sie keine Selbstverständlichkeit sei, vor allem für jene, die sich noch im Asylverfahren befinden. Es bestand eine gewisse Skepsis, durch detaillierte Äußerungen einen persönlichen Nachteil hinsichtlich ihres Aufenthalts in Österreich hinnehmen zu müssen. Eine Voraussetzung war daher, die Interviews unter dem Schutz der Anonymität zu führen. Dementsprechend wurden für die Interviewpartner die Pseudonyme Adam, Bob, Chuis, David und Eric ausgewählt. Es wurde auch versucht, mit nigerianischen Verkäuferinnen Interviews zu arrangieren, jedoch ohne Erfolg. Dem Anschein nach hatten sie eine deutlich höhere Hemmschwelle als ihre männlichen Kollegen. Bezüglich der Sprache war es jedem selbst überlassen, ob er sich besser bzw. lieber auf Deutsch oder Englisch ausdrücken wollte. Die Form der Interviews war möglichst frei und narrativ, d. h. die Interviewpartner wurden so wenig wie möglich unterbrochen. Teilweise waren die Gespräche zu Beginn von einer längeren Anlaufphase geprägt, aber es benötigt in dieser Art von Interview zu jedem Menschen einen anderen Zugang, um einen gewissen Redefluss herbeiführen zu können. Die Fragen waren nicht ausformuliert und die Themen nicht strikt vorgegeben. Eher war es das Ziel, biographisch zu arbeiten, um dann bei bestimmten Themen zu versuchen, intuitiv nachzuhaken. So sind im Großen und Ganzen überaus ergiebige Interviews mit einem breiten Themenspektrum entstanden, die für diese Arbeit, aber auch darüber hinaus, aufschlussreich sind. Auch mit der langjährigen Geschäftsführerin des 20ers, Sylvia Riedmann-Flatz, wurde ein qualitatives Expert_inneninterview geführt. Mit über einem Jahrzehnt Erfahrung in diesem Bereich konnte sie wertvolle Informationen geben, die in dieser Form sonst nirgends zu finden waren.

\section{Nigeria}

Als „Crippled Giant" bezeichnete Osaghae 1998 den Zustand seines Heimatlandes, welches seit der Unabhängigkeit von Großbritannien im Jahr 1960 eine turbulente Zeit hinter sich hat. Ethnische wie religiöse Spannungen, bewaffnete Konflikte, Misswirt-

\footnotetext{
6 Naika Foroutan, Postmigrantische Gesellschaften, in: Heinz Ulrich Brinkmann/Martina Sauer (Hrsg.), Einwanderungsgesellschaft Deutschland. Entwicklung und Stand der Integration, Wiesbaden 2016, S. 227-254.

7 Homi Bhabha, The Location of Culture, London-New York 1994

8 Osaghae, Crippled Giant.
} 
schaft, Korruption, Armut, Umweltverschmutzung und Militärdiktaturen waren und sind ständige Begleiter des bevölkerungsreichsten Landes Afrikas. ${ }^{9}$

Ökonomisch gesehen ist Nigeria seit 2014 zusammen mit Südafrika die größte Volkswirtschaft Afrikas. ${ }^{10}$ Trotz stets steigendem Bruttoinlandsprodukt steigt die Arbeitslosigkeit, vor allem die Jugendarbeitslosigkeit. Seit Anfang der 2000er wuchs sie deutlich auf über zehn, bis 2010 auf über zwanzig Prozent an. ${ }^{11}$ Gleichzeitig lebten 2010, nach den aktuellsten Daten der Weltbank, 85 Prozent der Bevölkerung unter der Armutsgrenze von zwei US-Dollar pro Tag. ${ }^{12}$ Die überaus junge Bevölkerung von 183 Millionen Menschen wächst schnell: In konkreten Zahlen bedeutet das ein Bevölkerungswachstum von 43 Millionen seit 2006. ${ }^{13}$ Eine Ursache für das rapide Bevölkerungswachstum ist das marode Bildungssystem, in dem vor allem die weibliche Bevölkerung benachteiligt ist. Es fehlt an Aufklärung und an Investitionen in Familienplanung. Verhütungsmittel werden nur in einem geringen Maß benützt. ${ }^{14}$ Das bringt einen hohen Bevölkerungsdruck und Verteilungsprobleme mit sich. Prognosen zufolge wird Nigeria bis 2050 nach Indien und China das Land mit der drittgrößten Bevölkerung weltweit sein. ${ }^{15}$ Da somit die Perspektiven immer kleiner werden, suchen viele ihr Glück woanders und wandern aus.

Die Entwicklung des Landes seit der Unabhängigkeit charakterisiert Kappel als "growth without development" ${ }^{\prime 16}$. Das hätte nicht so kommen müssen, denn die Voraussetzungen für Wohlstand und Prosperität waren gegeben: Große Vorkommen an Bodenschätzen im sogenannten "Middle Belt", reiche Ölvorkommen im Nigerdelta sowie ein enormes landwirtschaftliches Potential versprachen eine rosige Zukunft. ${ }^{17}$ Die Gründe für das Scheitern sind vielfältig und bedingen sich gegenseitig. Diese Faktoren hatten großen Einfluss auf die Kultur(en) und Identität(en) Nigerias und sind gleichzeitig auch Beweggründe, das Land zu verlassen. Um die Gegenwart besser zu verstehen, werden daher die wichtigsten Entwicklungen in der Geschichte Nigerias seit dem Beginn des 19. Jahrhunderts nachgezeichnet.

9 Bundesministerium für wirtschaftliche Zusammenarbeit und Entwicklung, Nigeria. Situation und Zusammenarbeit, o.D., [http://www.bmz.de/de/laender_regionen/subsahara/nigeria/zusammenarbeit/index.html], eingesehen 21.3.2018

10 BBC online (Hrsg.), Nigeria becomes Africa's biggest economy, 6.4.2014, [http://www.bbc.com/news/ business-26913497], eingesehen 10.6.2017.

11 Ditimi Amassoma/Philip Ifeakachukwu Nwosa, The Impact of Unemployment Rate on Productivity Growth in Nigeria: An Error Correcting Modeling Approach, in: International Journal of Economics and Management Services 2 (2013), Heft 8, S. 1-13, hier S. 7, [https://www.omicsonline.com/open-access/the-impact-of-unemploymentrate-on-productivity-growth-in-nigeria-an-error-correction-modeling-approach-2162-6359-2-145.pdf], eingesehen 11.6.2017.

12 Reiner Klingholz/Felix Lill/Joachim Budde, Wachsen und Schrumpfen. Nigeria: Außer Kontrolle, in: Zeit Online (2014, [http://www.zeit.de/2014/07/demografie-iran-nigeria-japan-deutschland/seite-2], eingesehen 29.8.2017.

13 Seit 2006 erfolgte bei einer Lebenserwartung von 54 Jahren ein Bevölkerungswachstum von 2,3 \%, die Fertilitätsrate betrug dabei 5,5 Kinder pro Frau. Den größten Anteil an der Bevölkerung haben mit Abstand die 15- bis 19-Jährigen: National Bureau of Statistics Nigeria (Hrsg.), 2015 Statistical Report on Women and Men in Nigeria, o. O. (Abuja) 2016, S. 1-5, [http://www.nigerianstat.gov.ng/download/491], eingesehen 10.6.2017.

14 Klingholz/Lill/Budde, Wachsen und Schrumpfen, eingesehen 29.8.2017.

15 Ebd.

16 Kappel, Nigeria, S. 141.

17 Falola/Heaton, A History of Nigeria, S. 2-3. 


\subsection{Nigeria von 1800 bis 1960}

In den Jahren zwischen 1500 und 1800 wuchs der Sklav_innenhandel auf dem Gebiet des heutigen Nigeria zu einer wirtschaftstragenden Institution heran. ${ }^{18}$ Nicht nur mit den Europäer_innen, sondern auch innerhalb der dortigen westafrikanischen Gesellschaften spielten Sklav_innen eine wichtige Rolle in den ökonomischen Kreisläufen. In den nördlichen Savannengebieten, wo der Sklav_innenhandel bereits vor 1500 Tradition hatte, waren das islamische Reich Borno, das sich um den Tschadsee herum erstreckte, sowie die westlich davon gelegenen kleineren Hausa-Staaten die Hauptakteure. ${ }^{19}$ Im Süden transferierte insbesondere das Königreich Oyo die Sklav_innen zu den europäischen Haupthandelsstützpunkten der Europäer_innen in den Buchten von Benin und Biafra. ${ }^{20}$ Damals wie heute lebten auf dem Gebiet Nigerias zahlreiche kleinere und einige größere ethnische Gruppierungen. Noch heute sprechen in Nigeria über 350 verschiedene Ethnien etwa vierhundert Sprachen und Dialekte. ${ }^{21}$ Zu Beginn des 19. Jahrhunderts kam es sowohl zu massiven politischen und kulturellen als auch zu ökonomischen Umwälzungen. Grundsätzlich verlief die Entwicklung im Norden unter anderen Vorzeichen als im Süden. Es manifestierten sich Unterschiede, die - wie noch ausgeführt werden wird - auf verschiedensten Ebenen bis heute immer wieder zum Tragen kamen.

Der Konflikt zwischen (christlichen) Southerners und (muslimischen) Northerners war und ist eines der Hauptprobleme Nigerias. Bereits lange vor der britischen Kolonialherrschaft haben sich Handels- und damit auch Migrationswege von Nordafrika durch die Sahara hindurch etabliert. ${ }^{22}$ Im nördlichen Gebiet des heutigen Nigeria war der Islam daher bereits im 11. Jahrhundert angekommen, jedoch nicht flächendeckend mit Ausnahme von Borno im Nordosten des heutigen Staatsgebietes. Im Zuge des Jihads von Usman Dan Fodio im Jahr 1812 entstand mit dem Kalifat Sokoto im Norden ein expansiver theokratischer Staat, der fast die gesamten nördlichen Hausa-Gebiete umfasste. ${ }^{23}$ In den nächsten Jahrzehnten expandierte Sokoto nach Osten, Südosten und Süden. ${ }^{24}$ Dieser Jihad ist als soziale und politische Reaktion auf Korruption und Unterdrückung zu sehen, aber auch vor dem Hintergrund, anstatt dem mit traditionellen Religionen vermischten einen frommen Islam nach arabischem Vorbild einzuführen. ${ }^{25}$ Das gelang nicht vollständig, veränderte die Gesellschaft aber nachhaltig: Die Scharia wurde zur Gesetzesgrundlage, islamische Architektur, islamisches Liedgut sowie islamische Lyrik zählten zum neuen kulturellen Fundament. ${ }^{26}$ Durch die flächendeckende Einführung von Koranschulen bekamen nun breite Massen Zugang zu Bildung, was vormals nur einer kleinen städtischen Minderheit vorbehalten war. ${ }^{27}$ Dan Fodios Reformen erfreuten

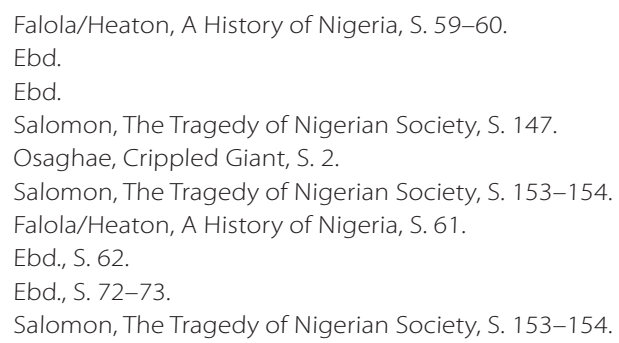


sich zunehmender Beliebtheit und bis zum Beginn des 20. Jahrhunderts identifizierte sich der überwiegende Teil der Einwohner_innen Sokotos mit dem Islam. ${ }^{28}$

Im Süden vollzog sich ein politischer, wirtschaftlicher sowie kultureller und religiöser Wandel. Westlich des Nigers existierte eine Ansammlung von Staaten, Königreichen und anderen kleineren Gesellschaften, wobei die Königreiche Benin und Oyo die maßgebenden Akteure waren. ${ }^{29}$ Im Osten waren - im Gegensatz zum Westen - dezentral organisierte Gesellschaften und Stadtstaaten wie Calabar oder Opobo angesiedelt. ${ }^{30}$ Der Südwesten war in der Zeit von 1790 bis etwa 1830 vom Zerfall des Oyo-Reiches geprägt. ${ }^{31}$ Zum einen kam es zu inneren Revolten und Abspaltungen, aber auch die nördlichen Nachbarn annektierten große Gebiete von Oyo. Sokoto expandierte weiter nach Süden und breitete damit auch den islamischen Jihad aus. Durch innere Rebellionen wurde es immer schwieriger, die Routen für den Sklavenhandel zu kontrollieren. Mit dem zunehmenden Zerfall entstand ein Machtvakuum und durch die darauffolgenden Binnenfluchtbewegungen etablierten sich neue Machtzentren. ${ }^{32}$

Zum anderen wurde der Sklavenhandel von den Briten 1807 offiziell aufgegeben, wiewohl von dessen wirklich weitgehendem Ende erst Mitte des 19. Jahrhunderts gesprochen werden kann. ${ }^{33}$ Darüber hinaus hat sich dadurch die kulturelle Landschaft verändert, indem verschiedene Kulturen - soziale Normen und Rechtspraxen - ineinander übergegangen sind. Neben diesen internen Vorgängen wirkte sich der zusehends größer werdende britische Einfluss aus, was unter anderem eine gewisse politische Stabilität brachte. Mitte des 19. Jahrhunderts stiegen - vom südwestlich gelegenen Lagos und vom Nigerdelta ausgehend - die christlichen Missionierungsbemühungen an. Damit einhergehend errichteten die Briten neben Kirchen und Missionierungsgebäuden landesweit westlich geprägte Schulen. ${ }^{34}$ Englisch wurde zur Schul- und Amtssprache, die wirtschaftlichen Beziehungen zwischen Briten und der ansässigen Bevölkerung wurden zunehmend enger. ${ }^{35}$ Die traditionellen Strukturen verloren langsam an Bedeutung und es war für die Einheimischen erstrebenswert, zum Christentum zu konvertieren sowie sich der westlichen Kultur anzupassen. Damit wurde sozialer Aufstieg möglich und der einheimischen Bevölkerung stand dadurch die „Welt des weißen Mannes" offen bzw. offener. ${ }^{36}$

In einem Prozess, der knapp ein halbes Jahrhundert dauerte, wurde der südliche Teil des heutigen Nigeria Anfang des 19. Jahrhunderts aus wirtschaftlichen Interessen und in Konkurrenz zur deutschen und französischen Kolonialpolitik zum britischen Protektorat erklärt. ${ }^{37}$ Zur gleichen Zeit wurden die Kalifate Sokoto und Borno gewaltsam ok-

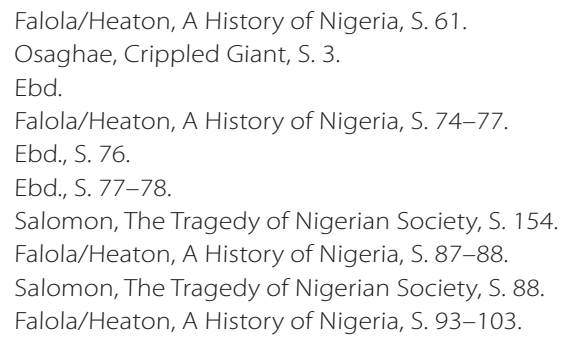


kupiert, um den französischen Absichten im Norden entgegenzuwirken. ${ }^{38}$ Somit wandelten sich die Gebiete im Norden sowie im Süden bis zu Beginn des 19. Jahrhunderts: Aus einem Konglomerat verschiedener unabhängiger und lose verbundener Territorien wurden zwei etwas homogenere, aber bei weitem nicht einheitliche Großräume. ${ }^{39}$ Die beiden Protektorate wurden 1914 aus wirtschaftlich-administrativen Gründen zu einer Kolonie vereinigt. ${ }^{40}$ Dennoch entwickelten sie sich auf unterschiedliche Weise: Während der Süden eher direkt regiert wurde und unter westlichen Einfluss stehende Gebildete die neuen Eliten stellten, erfolgte im Norden die Machtausübung mittels Indirect Rule, d. h. die bisherigen traditionellen Strukturen des Kalifats wurden aufrechterhalten und die dortigen konservativen Eliten blieben an der Macht. ${ }^{41}$ Das bedeutete, dass der überwiegende Teil des Nordens nach wie vor von der westlichen Kultur und damit auch von westlicher Bildung und christlicher Missionierung weitgehend abgeschirmt blieb. ${ }^{42}$

Nichtsdestotrotz wurden etwa die Infrastruktur, Institutionen und Bürokratie angeglichen, das Eisenbahnsystem vereinheitlicht sowie eine gemeinsame Währung eingeführt. ${ }^{43}$ Dadurch verloren die alten transsaharischen Handelswege zugunsten des Binnenhandels an Bedeutung. ${ }^{44}$ In einem fließenden Übergang bis etwa 1850 ersetzte vor allem die Palmölproduktion den Sklavenhandel. Generell wurden die traditionellen Vertriebssysteme innerhalb weniger Jahre durch eine kapitalistische Exportwirtschaft auf Basis von Bodenschätzen und agrarischen Produkten ersetzt. ${ }^{45}$ Die wichtigsten Güter neben Palmöl waren Erdnüsse, Kakao, Baumwolle, außerdem Bodenschätze wie Zinn, Kohle, Gold, Silber oder Diamanten. ${ }^{46}$ Die politisch-ökonomischen Umwälzungen führten zu einem rasanten Städtewachstum, bedingt unter anderem durch neue Geschäftsmöglichkeiten und einem erhöhten Bedarf an Arbeitskräften. ${ }^{47}$ Die Haupthandelszentren lagen an der südlichen Küste. Die durch den Binnenhandel in den Norden gewanderten Handels- und Geschäftsleute hatten es leichter, in der Wirtschaft Fuß zu fassen als die im Norden ansässige Bevölkerung: Sie sprachen flüssiges Englisch, hatten eine westliche Bildung und zudem gute Kontakte zu ihren Familien zu Hause. ${ }^{48}$ Es darf jedoch in diesem Zusammenhang nicht vergessen werden, dass die koloniale Wirtschaft darauf ausgerichtet war, nutzbringend für die Kolonialmacht zu sein. Die Menschen wurden von größtenteils schlecht bezahlter Lohnarbeit abhängig gemacht, zudem positionierten sich große europäische Firmen, gegen die etwa nigerianische Kleinbauern nicht ernsthaft konkurrieren konnten. ${ }^{49}$ Dennoch, bis in die 1960er-Jahre waren Agrarprodukte eine der Haupteinnahmequellen Nigerias. Das Land war der

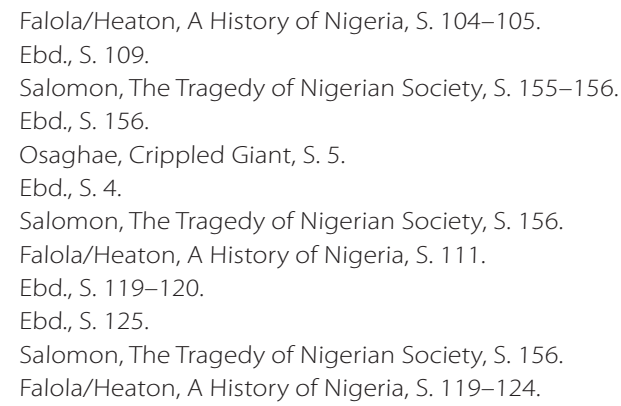


weltweit größte Palmölproduzent und auch der Export von Kakao, Gummi, Erdnüssen sowie Bodenschätzen lag international im Spitzenfeld. ${ }^{50}$ Die ökonomischen Maßnahmen wie etwa die Transformation der traditionellen in eine kapitalistische Exportwirtschaft führten zu einer engeren Verzahnung von Nord und Süd. Abseits der Wirtschaft setzte sich die unterschiedliche Entwicklung jedoch fort. Vor allem im Bereich der Bildung herrschten Differenzen: Während im Süden z. B. 1912 schon 150 Primärschulen existierten, betrug die Anzahl im Norden nur 34. Diese Kluft zog sich bis zum universitären Sektor durch und führte dazu, dass sich um 1950, kurz vor der Unabhängigkeit, hunderte Akademiker aus dem Süden einem einzigen aus dem Norden gegenüberstanden. ${ }^{51}$ Diese massiven strukturellen Unterschiede waren auch in Bereichen der Modernisierung festzustellen. Der Norden hinkte dem Süden sowohl qualitativ als auch quantitativ um Jahre hinterher. Beispielsweise gab es in Lagos bereits 1890 die erste Zeitung, im Norden dauerte es noch bis 1935. Im Jahr 1950 erschienen im Süden 35, im Norden dagegen fünf Zeitungen. ${ }^{52}$

Politisch gesehen lief es ähnlich: Im Süden formierten sich politische Nationalparteien, deren Vertreter in die Institutionen sowie legislativen Vertretungen strömten, während die Situation in dieser Hinsicht im Norden bis Ende der 1940er-Jahre unverändert blieb. ${ }^{53}$ Das hat aber nicht nur mit westlicher Bildung zu tun, sondern auch mit der Indirect Rule im Norden und dem direkteren Einfluss der Briten im Süden, wo durch deren Präsenz die Forderung nach politischer Mitsprache immer lauter wurde. Von Beginn an gab es Widerstand gegen die britische Kolonialherrschaft, ab den 1930er-Jahren entstand daraus eine politische Bewegung, die hauptsächlich von europäisch gebildeten Intellektuellen getragen wurde. ${ }^{54}$ Im Gegensatz zum Norden hatten die Politiker im Süden zu diesem Zeitpunkt schon nennenswerte Erfahrungen in der zentralen legislativen Vertretung. Letztendlich manifestierten sich die Unterschiede der beiden Regionen im Jahrzehnt vor der Unabhängigkeit auf fast allen Ebenen. Aber nicht nur ein Nord-Süd-Gegensatz flammte auf, sondern auch der Gegensatz beider größten ethnischen Gruppen des Südens, zwischen Yoruba und Igbo. ${ }^{55}$ Insgesamt konnten entscheidende Machtpositionen im unabhängigen Staat zum größten Teil von Vertretern aus dem Süden eingenommen werden. Im Norden wurde daher befürchtet, im Zuge dessen vom Süden dominiert zu werden.

Diese Furcht wurde Anfang der 1950er politisch kanalisiert und als Konsequenz wurde die Politik der sogenannten Northernization betrieben. Das heißt, dass die nördlichen Eliten, allen voran die Gruppe der Hausa-Fulani, eine Zweiklassenpolitik betrieben. ${ }^{56}$ Die ansässige Bevölkerung des Nordens wurde gegenüber den zugezogenen Migrant_

50 Osaghae, Crippled Giant, S. 50.

51 Ebd., S. 5.

52 Ebd.

53 Ebd., S. 6.

54 Falola/Heaton, A History of Nigeria, S. 136

55 Thomas J. Davis/Azubike Kalu-Nwiwu, Education, Ethnicity and National Integration in the History of Nigeria: Continuing Problems of Africa's Colonial Legacy, in: The Journal of Negro History 86 (2001), Heft 1, S. 1-11, hier S. 1, [www.jstor.org/stable/1350175], eingesehen 16.6.2017. 
innen des Südens bevorzugt. Damit wurde beabsichtigt, Regierungsjobs zu ergattern und die Kontrolle über Produktion und Export zu gewinnen. ${ }^{57}$ Im Repräsentantenhaus wurde auf Basis der Einwohner_innenzahlen durchgesetzt, dass der Norden mindestens fünfzig Prozent der Sitze erhält (später 52 Prozent) und dass die gesamten Staatseinnahmen auch auf den wirtschaftlich schwächeren Norden verteilt werden. ${ }^{58}$ Diese Maßnahmen verhärteten die Fronten weiter und führten dazu, dass Politiker in erster Linie ihre Religion oder ihre Ethnie vertraten und nie größere Bevölkerungsschichten. Speziell auf regionaler und lokaler Ebene war dieser diskriminierende Regionalismus die Basis für eine Reihe zusätzlicher Spannungen, indem sich die verschiedenen Ethnien im Kampf um Privilegien in einem Nullsummenspiel gegeneinander aufrieben. ${ }^{59}$ Diesem Regionalismus wurde auch von der britischen Kolonialmacht zugearbeitet. Unter dem Stichwort einer natürlichen Spaltung Nigerias wurde schon 1939 der Süden auf Initiative des Gouverneurs Arthur Richards in eine östliche und eine westliche Provinz aufgeteilt sowie in weiterer Folge noch zusätzlich regionale Vertretungen eingeführt. ${ }^{60}$ Somit waren die noch heute bestehenden Konflikte und die Bruchlinien Nigerias am Vorabend der Unabhängigkeit bereits tief in der Gesellschaft verankert.

\subsection{Nigeria seit der Unabhängigkeit 1960}

Dieser uneinheitliche, fragmentierte Staat mit zahlreichen ethno-religiösen und regionalen Partikularinteressen machte eine Identifikation als Nigerianer_in nicht einfach. Aber es herrschte auch Hoffnung: Nach der Unabhängigkeit am 1. Oktober 1960 waren die Erwartungen groß. Eine vielversprechende Zukunft basierend auf wirtschaftlichen Erfolgen erschien realistisch, zudem wurden im Nigerdelta große Mengen an Erdöl gefunden. ${ }^{61}$ Mit dieser großen potentiellen ökonomischen Kraft wurde Nigeria anfangs vielfach noch "Giant of Africa"62 genannt. Die ökonomische Entwicklung war bis 1966 vielversprechend: Die Wirtschaft wuchs und durch eine angekurbelte Güterproduktion sowie die Förderung der Minen- und Erdölindustrie wurde ein Diversifizierungsprozess in die Wege geleitet. ${ }^{63}$

Auch auf kultureller Ebene, etwa durch literarische Werke oder Kunst, wurde versucht, eine gesamtnigerianische Identität, ein gemeinsames Bewusstsein aufzubauen. ${ }^{64}$ Das wurde ebenso mithilfe technischer Errungenschaften angestrebt und es schien möglich, sich dem Niveau der USA und Europa anzunähern, z. B. mit der Etablierung des ersten Fernsehsenders Afrikas oder dem Bau des damals hochmodernen Liberty Stadium in der südwestlichen Stadt Ibadan. ${ }^{65}$

\footnotetext{
57 Salomon, The Tragedy of Nigerian Society, S. 156-157.

58 Osaghae, Crippled Giant, S. 6.

59 Salomon, The Tragedy of Nigerian Society, S. 157-158.

60 Osaghae, Crippled Giant, S. 7.

61 Falola/Heaton, A History of Nigeria, S. 158.

62 Ebd.

63 Ebd., S. 165.

64 Ebd., S. 160-163.

65 Jacob Oluwole Odeyemi, A Political History of Nigeria and the Crisis of Ethnicity in Nation-Building, in: International Journal of Developing Societies 3 (2014), Heft 1, S. 87-95, hier S. 95, [https://doi.org/10.11634/216817831504459], eingesehen 20.6.2017.
} 
Eine Aufbruchsstimmung war also durchaus vorhanden, die Erreichung wirklicher Unabhängigkeit schien möglich. Letztendlich scheiterten diese ambitionierten Versuche jedoch daran, ein Nationalbewusstsein sowie eine prosperierende wirtschaftliche Entwicklung zu schaffen: "Everything looked possible - but everything went wrong.“66

Die Situation blieb verfahren: Die politischen Parteien waren aus dem Regionalismus der 1950er-Jahre geboren und vermochten keine integrative Politik zu betreiben. Nigeria war nur dem Namen nach eine Nation und die in der Kolonialzeit geschaffenen Strukturen erwiesen sich als äußerst resistent. Bis heute zieht sich auf allen gesellschaftlichen Ebenen ein rivalisierender und exklusiver Ethnozentrismus durch. ${ }^{67}$ Die Schaffung eines gesamtnigerianischen Bewusstseins wurde somit abgewürgt: „In many ways it was a state without a nation." ${ }^{68}$ Nach der Unabhängigkeit war Nigeria eine Föderation dreier relativ autonomer Regionen im Norden, Südwesten und Nordosten - jede davon hätte ein eigener unabhängiger Staat sein können. ${ }^{69}$ Der zentrale Begriff, der das Verhältnis dieser Regionen zueinander prägte, war Dominanz bzw. die Angst vor der Dominanz der jeweilig anderen. Wer in der zentralen Regierung und in den regionalen Vertretungen das Sagen hatte, bestimmte auch, wie die Ressourcen im Land verteilt werden sollen: Der im Parlament dominierende Norden fürchtete den weiter entwickelten Süden, umgekehrt der Süden den Norden und zusätzlich stand der Südwesten mit dem Südosten in Konkurrenz. ${ }^{70}$ Ferner fühlten sich die zahlreichen kleineren ethnischen Minderheiten bedroht und forderten, Nigeria in mehrere Staaten aufzuteilen. ${ }^{71}$ Als Konsequenz daraus wurde 1963 ein vierter Bundesstaat eingeführt, der vom Südwesten abgetrennt wurde: der Midwestern State. ${ }^{72}$

Bis in die Mitte der 1960er-Jahre schaukelte sich dieser unerbittliche Konflikt um die Vormachtstellung immer mehr auf, wodurch die erste Republik 1966 letztendlich zugrunde ging. Nach einem Militärputsch von Igbo-Offizieren aus dem Süden erfolgte ein Gegenputsch, angeführt von Militärangehörigen des Nordens, der in die Errichtung einer Militärdiktatur mündete. ${ }^{73}$ Das war nur der Beginn einer langen Reihe von Putschen, Gegenputschen und Putschversuchen. In weiterer Folge wurden zahlreiche im Norden lebende Igbo-Angehörige massakriert und eine Massenflucht in den Südosten setzte ein. ${ }^{74}$ Auf der Gegenseite wurden Northerners auf Igbo-Gebiet aus Rache und Vergeltung ermordet. ${ }^{75} 1967$ wurde versucht, das Minderheitenproblem durch die

66 Tom O'Neill, Curse of the Black Gold. Hope and betrayal on the Niger Delta, in: National Geographic online (2007), [http://ngm.nationalgeographic.com/2007/02/nigerian-oil/oneill-text], eingesehen 12.6.2017.

67 Marcel Ikechukwu Sunday Onyibor, National Identity and Crisis of Integration in Multi-Ethnic Nigeria: An Existentialist Perpective, in: Open Journal of Philosophy 6 (2016), S. 1-12, hier S. 4-7, [http://www.dx.doi. org/10.4236/ojpp.2016.61001], eingesehen 20.6.2017.

68 Folala/Heaton, A History of Nigeria, S. 159

69 Odeyemi, A Political History of Nigeria, S. 89

70 Falola/Heaton, A History of Nigeria, S. 165.

71 Osaghae, Crippled Giant, S. 36.

72 Lasse Heerten/A. Dirk Moses, The Nigeria-Biafra war: postcolonial conflict and the question of genocide, in: Journal of Genocide Research 16 (2014), Heft 2-3, S. 169-203, hier S. 172, [http://dx.doi.org/10.1080/14623528.201 4.936700], eingesehen 22.6.2017.

73 Heerten/Moses, The Nigeria-Biafra war, S. 173.

74 Ebd.

75 Osaghae, Crippled Giant, S. 63. 
Schaffung von zwölf Bundesstaaten (heute 36) - je sechs im Norden und im Süden zu lösen. ${ }^{76}$ Nach der Dreiteilung des Südostens waren die Igbo von den Ölvorkommen am Nigerdelta abgeschnitten. Unter anderem aus diesem Grund regte sich breiter Widerstand. ${ }^{77}$ Nach erfolglosen Verhandlungen über eine Unabhängigkeit des Südostens wurde 1967 die Republik Biafra ausgerufen, was zu einem blutigen Sezessionskrieg führte. ${ }^{78}$ Bis zum Ende des Krieges 1970 forderte er zwischen ein und drei Millionen Todesopfer. ${ }^{79}$ Der Biafra-Krieg ist nur ein trauriges Beispiel für die immer wieder ausufernde Gewalt, die das Land durchgehend bis heute schwer belastet.

Vor allem, wenn in den seltenen demokratischen Zeiten Wahlen bevorstanden, brachen immer wieder gewaltsame Konflikte aus. ${ }^{80}$ Bis zur Jahrtausendwende herrschten mit einigen wenigen Unterbrechungen fast ausschließlich Militärs. Politische Instabilität, Misswirtschaft und etliche Neuanfänge ließen das Land nie vollständig zur Ruhe kommen. ${ }^{81}$ Aufgrund der prekären Situation des Großteils der Bevölkerung wurden in den 1980ern die traditionellen religiösen Unterschiede immer mehr zu einem politisch aufgeladenen Identifikationsfaktor, was immer wieder zu Zusammenstößen zwischen Christen und Muslimen führte. ${ }^{82} 1993$ sollte ein jahrelang vorbereiteter Übergang zu einer zivilen Regierung erfolgen. Als sich der sozialdemokratische Kandidat Moshood Abiola im Juni des Jahres 1993 durchsetzte, annullierte der amtierende Präsident General Ibrahim Babadinga kurzerhand die Wahl. Er wollte die Macht nicht abgeben und stand zusätzlich unter dem Druck hoher Militärs und des konservativen Nordens. ${ }^{83}$ Damit schlitterte Nigeria abermals ins Chaos, das von Gewalt und Aufständen begleitet wurde - ein weiterer Bürgerkrieg stand unmittelbar bevor. ${ }^{84}$ Nach einer kurzen Übergangsphase - die als die Dritte Republik bezeichnet wird - stand Nigeria im November erneut unter Militärherrschaft. Der berüchtigte Diktator Sani Abacha regierte das Land mit harter Hand bis zu seinem Tod 1999. ${ }^{85}$ Zu diesem Zeitpunkt lebte die Bevölkerung Nigerias seit der Unabhängigkeit 38 Jahre unter Militärherrschaft. Nach Abacha wurde in der Vierten Republik die Demokratie wiedereingeführt, doch brachten diese Freiräume wiederum Probleme: Nachdem in den nördlichen Bundesstaaten die Scharia ganz oder teilweise eingeführt wurde, brachen erneut religiös motivierte, blutige Konflikte aus, die ein weiteres Mal zu zahlreichen Toten führten. ${ }^{86}$ Später, seit 2010, verbreitete die Terrororganisation Boko Haram vor allem im Nordosten Nigerias zusätzlich Angst

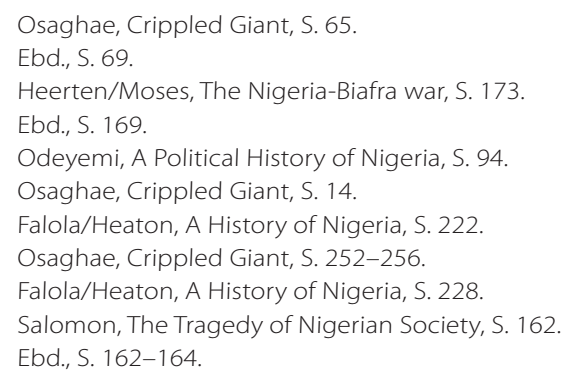


und Schrecken. ${ }^{87}$ Die Wahlen von 2015 weckten jedoch Hoffnung, denn erstmals erfolgte eine friedliche und demokratische Machtübergabe. ${ }^{88}$

Dass es über Jahrzehnte immer wieder zu Gewaltausbrüchen kam, liegt auch an der ökonomischen Entwicklung. Der wirtschaftliche Aufschwung kam mit dem Biafra-Krieg zu einem abrupten Ende. Ab Anfang der 1970er-Jahre wurde dann fast ausschließlich auf Erdöl gesetzt. Lag der Anteil von Erdöl an den Exporten 1969 noch bei etwa 40 Prozent, so hält er sich seit 1980 konstant bei mindestens 96 Prozent. ${ }^{89}$ Die anderen Wirtschaftszweige litten massiv darunter: Während in den 1960er-Jahren die Versorgung der eigenen Bevölkerung mit im Inland produzierten Nahrungsmitteln noch gewährleistet werden konnte, spielten der Industrie- sowie der ehemals große Landwirtschaftssektor ab den 1970ern keine Rolle mehr. ${ }^{90}$ Viele wollten am Öl-Boom teilhaben und dabei kam es zur Landflucht, welche in Verbindung mit politischem Missmanagement als bedeutender Faktor zum Niedergang des Agrarsektors beitrug. ${ }^{91}$ Insbesondere die Jugend, die in der Phase des Wirtschaftsaufschwungs noch im Bausektor Arbeit gefunden hatte, war davon betroffen. Infolge des Ölpreisverfalls Anfang der 1980erJahre standen sie dann ohne Perspektive da. ${ }^{92}$ Die Erdölindustrie konnte und kann nur relativ wenige Jobs schaffen: Das Rohöl wird exportiert, die wenigen Raffinerien im eigenen Land befinden sich in einem desolaten Zustand. Das führte dazu, dass Benzin und Diesel importiert werden mussten. ${ }^{93}$ Die Konzentration auf Erdöl brachte die Wirtschaft in einen Teufelskreis: Das Erdöl wertete die eigene Währung auf. Importe waren zwar billiger, andere Wirtschaftssektoren waren im internationalen Exportmarkt damit jedoch chancenlos. ${ }^{94}$

In die Infrastruktur wurde aus den Erdölgewinnen lediglich in der ersten Boomphase Anfang bis Mitte der 1970er-Jahre investiert, heute ist sie völlig marode. ${ }^{95}$ Beispielsweise wird die Abkürzung der National Electricity Power Agency (NEPA) sarkastisch "Not Expect Power Again"96 genannt. Rund um die Ölquellen entstanden vielschichtige Konflikte ${ }^{97}$ : Der Norden forderte einen Anteil der Gewinne, wogegen sich der Süden wehrte. Parallel dazu entwickelte sich ein Konflikt zwischen Regionalvertretungen und Zentralregierung um die zu verteilenden Ressourcen. Von den Öleinnahmen hatte die Bevölkerung praktisch nichts, diese gingen an Ölfirmen und an die Regierungen, welche häufig gemeinsame Sache mit den Konzernen machten. Im Gegenteil ist die

87 O. A., 'Boko Haram attack' frees hundreds of prisoners, in: BBC online, 8.9.2010, [http://www.bbc.com/news/worldafrica-11229740], eingesehen 22.6.2017.

88 Will Ross, Nigeria elections: Buhari scores historic win, in: BBC online, 1.4.2015, [http://www.bbc.com/news/worldafrica-32141703], eingesehen 22.6.2017.

89 Kappel, Nigeria, S. 144

90 Ebd., S. 145.

91 Salomon, The Tragedy of Nigerian Society, S. 59-60.

92 Ebd., S. 60.

93 Reiner Klingholz, Sklaven des Wachstums. Die Geschichte einer Befreiung, Frankfurt am Main-New York 2014, S. 234.

94 Kappel, Nigeria, S. 145.

95 Ebd., S. 149.

96 Salomon, The Tragedy of Nigerian Society, S. 164

97 Kappel, Nigeria, S. 157-160. 
Armut sehr hoch, die Umwelt weiträumig zerstört und das Trinkwasser trotz Wasserreichtums knapp. Widerstandsgruppen liefern sich Kämpfe mit den Ölfirmen und der Exekutive in einer "Kultur der Straflosigkeit"98 für letztere.

Insbesondere die Bereiche Wirtschaft, Politik und Verwaltung sind von einer extrem überbordenden Korruption geprägt, die schon immer eines der größten Probleme für Nigerias Entwicklung war. Laut dem Korruptionsindex von Transparency International rangierte Nigeria 2016 weit hinten auf Platz 136 unter allen Nationen. ${ }^{99}$ Bereits in der Kolonialzeit sind Fälle von Selbstbereicherung und Missbrauch öffentlicher Gelder überliefert. ${ }^{100}$ Nach der Unabhängigkeit wurde die Korruption allerdings nach und nach auf allen Ebenen institutionalisiert. Eine Besserung der Lage war und ist kaum in Sicht. Sowohl die Zivilregierungen als auch die Militärregimes bedienten sich in großem Umfang an den (Erdöl-)Ressourcen des Landes. ${ }^{101}$ Die zweifellos hohen Einnahmen aus dem Export kamen so gut wie nie dem Allgemeinwohl zugute - eine sehr kleine Oberschicht steht damit in krassem Gegensatz zur überwiegend armen Bevölkerung. So wurden z. B. in den 1970er-Jahren in ganz Nigeria zahlreiche industrielle Großprojekte initiiert, viele davon gingen jedoch nie in Betrieb. Solche sogenannten White Elephants dienten hauptsächlich dem Zweck, korrupte Politiker und Geschäftsleute mit einem regelrechten Raub an öffentlichen Geldern noch reicher zu machen. ${ }^{102}$ Oft wurde von den Regierungen angekündigt, die Korruption einzudämmen. Die anfangs meist populären Maßnahmen erwiesen sich jedoch im Nachhinein als halbherzig und äußerst ineffizient. ${ }^{103}$ Die Korruption fesselt Wirtschaft und Gesellschaft, das Vertrauen in staatliche Institutionen ist niedrig und mit der Demokratie - die in Wahrheit eine Kleptokratie ist - wurden die Probleme bis dato nicht wirklich weniger.

\subsection{Nigeria aus Sicht der interviewten Personen}

Die Erfahrungen der Interviewpartner in ihrem Heimatland fügen sich nahtlos in die hier geschilderte Darstellung über Nigeria ein. Es zeigte sich, dass sich vieles von der dortigen langjährigen Dauermisere in ihren Ausführungen wiederfindet.

Zunächst einige biographische Daten der interviewten Personen, die allesamt männlich und christlich sind: Adam und David stammen aus dem Südosten Nigerias, Bob und Eric aus dem Südwesten und Chuis, ursprünglich aus Ruanda, wuchs ab dem fünften Lebensjahr im Norden Nigerias auf. Alle Interviewpartner sind über dreißig Jahre alt und haben somit auch das besonders repressive Abacha-Regime bewusst miterlebt.

\footnotetext{
98 Kappel, Nigeria, S. 159.

99 Transparency International (Hrsg.), Corruption Perceptions Index 2016, 25.1.2016, [https://www.transparency.org/ news/feature/corruption_perceptions_index_2016], eingesehen 16.7.2017.

100 Michael M. Ogbeidi, Political Leadership and Corruption in Nigeria Since 1960: A Socio-economic Analysis, in: Journal of Nigeria Studies 1 (2012), Heft 2, S. 1-25, hier S. 6, [www.unh.edu/nigerianstudies/articles/.../Political_ leadership.pdf], eingesehen 25.6.2017.

101 Olu Awofeso/Temitayo Isaac Odeyemi, The Impact of Political Leadership and Corruption on Nigeria's Development since Independence, in: Journal of Sustainable Development 7 (2014), Heft 5, S. 240-253, hier S. 248-250, [http://dx.doi.org/10.5539/jsd.v7n5p240], eingesehen 25.6.2017.

103 Awofeso/Odeyemi, The Impact of Political Leadership, S. 249-250.
} 
Adam, Bob und Eric wuchsen in einer mindestens sechsköpfigen Familie auf - letzterer sogar in einer 23-köpfigen. Bob und David hatten Probleme mit den traditionellen Familienstrukturen, wodurch sie zu einer Binnenmigration gezwungen wurden. Generell war auffallend, dass alle bereits eine zum Teil mehrfache Migrationsgeschichte innerhalb Nigerias hinter sich hatten.

Im Bereich der Bildung hatte es niemand von den Befragten leicht, da Bildung in Nigeria meist nicht kostenlos ist und mit einer Ausnahme gaben alle an, als Kinder schon gearbeitet zu haben. Zwei der Interviewpartner wuchsen gänzlich ohne Schulbildung auf: Adam war lange Zeit ein Straßenkind in Lagos und Chuis arbeitete als Hirte im agrarisch geprägten Norden. David war nach der Sekundarstufe im Straßenbau tätig. Eric war als Soldat beschäftigt und schloss sich später - nach Problemen beim Militär - militanten Umweltschützern im Nigerdelta an. Bob hat ein Architekturstudium als Bachelor abgeschlossen und arbeitete unter anderem für eine deutsche Baufirma. Jeder der Befragten musste immer wieder mit widrigen Umständen, Armut, gefährlichen Situationen oder persönlichen Schicksalsschlägen zurechtkommen.

Alle Interviewpartner machten den Eindruck, gut über die Probleme Nigerias - sowohl in aktueller als auch in historischer Sicht - und über den potentiellen Reichtum des Landes Bescheid zu wissen. Ausführlich zur Sprache kamen vor allem die ethnischen und religiösen Konflikte. Adam ist ein Anhänger eines unabhängigen Biafras, er sagt: „We want our own freedom. We want that we have our own Biafra, this is why we are fighting."104 Er kritisiert - wie auch alle anderen Interviewpartner - den muslimischen Norden für eine zum Teil extreme religiöse Auslegung. Dieser Nord-Süd-Konflikt tritt in sämtlichen Interviews deutlich hervor. Aus der Sicht vieler Biafra-Anhänger heißt das: "[You] can't keep Christian and Muslim together, it cannot work." ${ }^{05}$ Jeder der Interviewten differenziert jedoch und spricht sich nicht per se gegen den Islam aus. Bob erzählt, dass es genauso strenge christliche Auslegungen gibt: „A Christian wouldn't drink beer [...], [and] don't eat pig in my country, especially the Catholics. [...] They take it to the extreme." ${ }^{106}$ Keine Bedeutung hatte Religion für Bob und Chuis, erst hier in Europa sind sie zu Christen geworden.

Die ethnischen und religiösen Konflikte in Nigeria sind nicht isoliert zu betrachten. In diesem Kontext spielen die Verteilungskämpfe im Zusammenhang mit der weitreichenden Armut, der grassierenden Kriminalität und Gewalt sowie der überbordenden Korruption eine große Rolle. Wie sich das lokal auf den Alltag auswirken kann, beschreibt Eric mit einem eindrücklichen Beispiel, in dem sich diese gesamte Problemkette erschließt:

„Some people eat from dustbin. If you watch some news, you will see little kids, they are stealing. [...] Because why? They are hungry. I watched the news, a boy was beaten because he stole garri. Garri is an African food. He stole it and he was beaten. Why? He's hungry, it is not his fault. But it is the consciousness

104 Tobias Leo, Interview mit Adam, Innsbruck, 29.6.2017, Aufnahme beim Verfasser, Anhang A, S. 45.

105 Leo, Interview mit Adam, S. 45.

106 Tobias Leo, Interview mit Bob, Innsbruck, 1.7.2017, Aufnahme beim Verfasser, Anhang B, S. 64. 
there, they [the government] are taking the money and nobody is there to fight with them." ${ }^{107}$

Wenn die nigerianische Regierung zur Sprache kam, fielen die Reaktionen durchaus negativ aus. "They are criminals. They are stealing everything we have. We have the sources, but our old problem in Nigeria is government"108, lautet der überwiegende Grundtenor. Ein Vertrauen in die Politik ist kaum wahrzunehmen, auch die Demokratie verbessere die Lage kaum: „In my country, we have a democratic government but, it's just in the book. It's not really as the same here [in Austria]." ${ }^{\prime 109}$

Die Sicherheitslage ist den Aussagen nach schlecht, Polizei und Militär seien Teil des Problems: „You can shoot someone, the police don't say anything, they don't give a fuck."110 Eric, der selbst acht Jahre in der Armee diente, erzählt: „So, in the military there, I'd never liked the way they were doing an oppression to people, because the military oppressed the civilians."111 Die über viele Jahre und Konflikte gewachsene Mentalität wurde ebenfalls thematisiert. "Our mind is already poisoned"112, konstatiert Bob und David stellt fest: "Das Problem von Afrika ist die Mentalität.."113

Insgesamt ergänzen die Aussagen der Befragten die an sich schon düstere Problemlage in Nigeria auf eine persönliche Weise und bestätigen diese auch. Es herrschte vor, in und nach den Gesprächen immer der Eindruck, dass den Interviewpartnern Nigeria zwar am Herzen liegt, durch die Verkettung von Problemen in Verbindung mit einem bestimmten Auslöser hatten sie aber einen guten Grund, auszuwandern.

\section{Auswanderung und Leben in Europa}

Nigeria ist zugleich Einwanderungs- und Auswanderungsland, wobei die Auswanderungsrate überwiegt. ${ }^{114}$ Beispielweise verließen 2012 und 2013 fast zwei Millionen Menschen das Land. In den Jahren zwischen 1990 und 2013 kam es dabei zu einigen signifikanten Veränderungen: Zunächst verdoppelte sich die Migration in diesem Zeitraum und während 1990 etwa ein Drittel der Auswanderung in wohlhabendere Regionen erfolgte, waren es 2013 bereits knapp zwei Drittel. Die wichtigsten Zielregionen sind (Stand 2013) andere afrikanische Staaten (35,6 Prozent), Europa (34,2 Prozent) und Nordamerika (26,4 Prozent). Die Migration nach Europa hat sich seit 1990 von knapp 90.000 auf etwa 350.000 Person im Jahr 2013 fast versechsfacht. ${ }^{115}$ Ein großer Teil der Auswanderer ist gut ausgebildet, in Europa liegt deren Anteil laut einer Analyse der International Organization for Migration (IOM) 2009 bei 46 Prozent. ${ }^{116}$ Es wird durch den

\footnotetext{
107 Tobias Leo, Interview mit Eric, Innsbruck, 23.8.2017, Aufnahme beim Verfasser, Anhang E, S. 94

108 Leo, Interview mit Adam, S. 44.

109 Leo, Interview mit Bob, S. 67.

110 Ebd., S. 65.

111 Leo, Interview mit Eric, S. 90.

112 Leo, Interview mit Bob, S. 64.

113 Tobias Leo, Interview mit David, Innsbruck, 12.7.2017, Aufnahme beim Verfasser, Anhang D, S. 83.

114 Uche C. Isiugo-Abanihe/IOM Nigeria, Migration in Nigeria. A Country Profile 2014, Genf 2016, S. 86, [https:// publications.iom.int/system/files/pdf/mp_nigeria.pdf], eingesehen 26.6.2017.

115 Isiugo-Abanihe/IOM, Migration in Nigeria, S. 109

116 Ebd., S. 32
} 
Mangel an (vergleichbaren) Daten geschätzt, dass die meisten männlich, zwischen 18 und 35 Jahre alt und in den Bereichen Ingenieurswesen, Medizin, Bildung, Rechtswissenschaften und Informationstechnologie spezialisiert sind. ${ }^{117}$

Die Abwanderung von hochqualifizierten Kräften bedeutet für Nigeria zunächst ein Brain Drain, also einen Verlust von geistigen Ressourcen, der für Schlüsselsektoren wie Bildung oder Gesundheit negative Konsequenzen mit sich zieht. Das muss aber nicht so bleiben: Wenn auch niedrig qualifizierte Personen z. B. in westlichen Industrieländern Fähigkeiten erwerben und gegebenenfalls dann nach Nigeria zurückkehren (müssen), kann das durchaus zu einem Brain Gain führen, d. h. die mitgebrachten Qualifikationen und Fertigkeiten wirken sich positiv aus. Für Europa bedeutet die Zuwanderung vieler gebildeter Nigerianer_innen ebenfalls ein potentielles Brain Gain. Jedoch besteht auf beiden Seiten die Gefahr des Brain Waste, falls dieses Potential nicht genutzt wird, etwa wenn jemand seine erworbene Qualifikation nicht ausüben kann oder darf. Reguläre Arbeitsmigration ist durch restriktive Zugangsregeln zu den EU-Arbeitsmärkten eher die Ausnahme. ${ }^{118}$

Entsprechend dem europäischen Gesamtbild stieg die Anzahl nigerianischer Migrant_ innen auch in Österreich: Von 3.664 in Nigeria geborenen Personen Anfang 2002 hat sich die Zahl zu Jahresbeginn 2017 auf 8.879 erhöht. ${ }^{119}$ Unter den Nationen Afrikas stellen Nigerianer_innen damit die am meisten in Österreich vertretene Gruppe von Zugewanderten. Mit 4.488 Personen lebte Anfang 2017 der Großteil in Wien. In Tirol haben sich 420 Personen niedergelassen und sind nach den Somalier_innen die zweitgrößte Gruppe von Afrikaner_innen in Tirol. ${ }^{120}$

\subsection{Die Wege der Interviewpartner nach Tirol}

Zunächst ist festzuhalten, dass keiner der Interviewpartner Österreich, geschweige denn Tirol, gekannt hatte. Deutschland war ihnen schon eher ein Begriff und zum Teil auch Ziel. Die Gründe bzw. Anlässe für die Auswanderung aus Nigeria waren bei allen verschieden, eine gewisse Perspektivlosigkeit war jedoch immer ein Teil davon. Klassisches Beispiel für Armutsmigration ist Adam, der sich damals dachte: "I don't have anything to do in Nigeria, maybe Europe would be better."121 Bob war ursprünglich nach Libyen migriert, um besser zu verdienen. Er arbeitete auf einem Schiff von Agip und den Entschluss nach Europa zu gehen, fasste er spontan. Als er sich unerlaubt auf das italienische Festland begab, um Rom zu sehen, traf er die Entscheidung, in Europa zu bleiben: „I didn't wanna go back to Africa, I didn't wanna go back to Libya."122 Drei

117 Isiugo-Abanihe/IOM, Migration in Nigeria, S. 33.

118 Andreas Baumer, Irreguläre Migration und staatliche Politik in Spanien und Europa, Wiesbaden 2017, S. 17.

119 Statistik Austria, Bevölkerung zu Jahresbeginn 2002-2017 nach detaillierter Staatsangehörigkeit, 23.5.2017, [http://www.statistik.at/wcm/idc/idcplg?ldcService=GET_PDF_FILE\&RevisionSelectionMethod=LatestReleased \&dDocName=037044], eingesehen 7.7.2017.

120 Statistik Austria, Bevölkerung am 1.1.2017 nach detailliertem Geburtsland und Bundesland, 23.5.2017, [http:// www.statistik.at/wcm/idc/idcplg?IdcService=GET_PDF_FILE\&RevisionSelectionMethod=LatestReleased\&dDocN ame=023841], eingesehen 7.7.2017.

121 Leo, Interview mit Adam, S. 38.

122 Leo, Interview mit Bob, S. 57. 
der Interviewpartner flüchteten, weil sie konkret in Lebensgefahr schwebten: Chuis entkam der Gefangenschaft von Boko Haram, David hatte ein Problem mit den traditionellen Familienstrukturen, die in den vorchristlichen Igbo-Religionen eingebettet sind. Er beschrieb zwar keine Details, deutete aber wohl auf traditionelle Opferrituale hin, in denen mitunter auch Menschenopfer enthalten sein können. ${ }^{123}$ Eric hatte nie die Absicht, nach Europa zu kommen, da er als Soldat ein gutes Leben führte. Aufgrund eines religiös-ethnischen Konflikts innerhalb des Militärs befand er sich plötzlich in einer bedrohlichen Situation: Nach dem Verlust seiner Waffe, was als ein schweres Vergehen angesehen wird, schloss er sich zunächst den Rebellen im Nigerdelta an, die gegen die dortige Umweltverschmutzung paramilitärisch vorgehen. Den Rebellen wurde zur Beruhigung der Lage eine Generalamnestie gewährt, die aber wieder aufgehoben wurde. Aufgrund dessen nahm das Militär wieder seine Verfolgung auf und daraufhin entschloss er sich zur Flucht.

Adam, Bob und Chuis erzählen, dass sie auf ihrer Route nach Europa in Libyen nicht mehr vorankamen und für das nötige Geld betteln bzw. arbeiten mussten. Alle drei überquerten danach mit einem Schiff das Mittelmeer. David macht dazu keine Angaben, jedoch sind er und sein Vater mithilfe eines katholischen Missionars nach Europa gekommen. Eric landete mit dem Flugzeug in Wien. Generell war Wien die erste Station für alle Interviewten in Österreich. Nach Tirol gelangten sie erst später aufgrund von Verteilungsmaßnahmen der zuständigen Behörden, d. h. niemand der Befragten kam über die Brennerroute nach Tirol. Bob, Chuis, David und Eric erreichten Österreich in den Jahren zwischen 2009 und 2013. Adam gibt an, bereits seit etwa 15 Jahren hier zu sein. Die Interviews zeigen sowohl Gemeinsamkeiten als auch individuelle Unterschiede in den Migrationsrouten sowie in den Anlässen, die letztendlich zur Auswanderung führten. Klar ist, dass es nie den einen Grund zur Auswanderung gibt, aber auch, dass Armut und Perspektivenlosigkeit dabei stets eine Rolle spielen. Was niemand von den Interviewpartnern wusste, ist, was eigentlich in Europa legal oder illegal ist und v. a. war ihnen der Asylbegriff nicht bekannt. Die Chance, Asyl im Sinne der Genfer Flüchtlingskonvention zu bekommen, dürften allerdings nur Chuis und Eric haben.

\subsection{Asylchancen für Nigerianer_innen in Österreich}

Aus den Statistiken geht hervor, dass Nigerianer_innen hauptsächlich über den Weg des Asylverfahrens versuchen, sich dauerhaft in Österreich niederzulassen. Österreichweit gab es im Zeitraum von 2005 bis 2015 durchschnittlich etwa 650 Asylanträge pro Jahr. ${ }^{124}$ Laut dem österreichischen Innenministerium war Nigeria mindestens seit 2002 immer unter den zehn antragsstärksten Nationen, letztendlich mit wenig Erfolg. ${ }^{125}$ Die

123 Nworgu Sharon Ifeoma, Living sacrifice among the Igbo, in: The Guardian online (2015), [https://guardian.ng/ sunday-magazine/c180-arts/living-sacrifice-among-the-igbo/], eingesehen 25.11.2017.

124 Statistik Austria, Statistisches Jahrbuch 2017, Wien 2017, S. 113, [https://www.statistik.at/wcm/idc/ idcplg?ldcService=GET_PDF_FILE\&dDocName=054401], eingesehen 7.7.2017.

125 Bundesministerium für Inneres Österreich, Statistiken Asylwesen, O. D., [http://www.bmi.gv.at/cms/bmi_ asylwesen/statistik/start.aspx], eingesehen 22.7.2017. 
Chance auf Asyl ist sehr gering: Von 2005 bis 2015 gab es im Durchschnitt nicht ganz 13 positive Asylentscheidungen pro Jahr. ${ }^{126}$ Das entspricht einer Quote von etwa zwei Prozent. Nicht herauszulesen sind in diesen Statistiken jedoch die verschiedenen Instanzenwege. Somit sind fast alle Nigerianer_innen von der Abschiebung bedroht. Nigeria zählt in Österreich nicht zu den sicheren Herkunftsländern. ${ }^{127}$ In verschiedenen Urteilen des Bundesverwaltungsgerichtshofs geht jedoch hervor:

„Auch wenn die Lage in Nigeria regional instabil ist, kann im Sinne der maßgeblichen Rechtsprechung keineswegs von einer realen Gefahr der Verletzung von Bestimmungen der EMRK für Rückkehrer nach Nigeria schlechthin - etwa aufgrund eines Bürgerkrieges oder einer Hungersnot - gesprochen werden." ${ }^{128}$

Diese Erkenntnis legt nahe, dass in der Rechtssprechungspraxis Nigeria sehr wohl als sicheres Herkunftsland betrachtet wird, was bedeutet, dass viele abgeschoben werden. Die Dauer der Asylverfahren kann sich jedoch über viele Jahre erstrecken. Ein Extrembeispiel kam 2014 an die Öffentlichkeit, wonach ein Mann aus Bangladesch selbst nach 18 Jahren Wartezeit noch keinen Bescheid in letzter Instanz erhalten hatte. ${ }^{129}$ Auch die für diese Arbeit interviewten Personen berichten von langjährigen Wartezeiten von bis zu acht Jahren. Fast ein Jahrzehnt in unsicheren und oft prekären Verhältnissen zu leben ist also durchaus keine Seltenheit.

\subsection{Integration und Arbeitsmarktsituation für Asy/werber_innen in Österreich}

Drittstaatsangehörige werden seit 2002 durch die Integrationsvereinbarung verpflichtet, sich (vor allem sprachlich) zu integrieren. ${ }^{130}$ Jene umfasst zwei Module, wobei das erste verpflichtend und mit Sanktionen verbunden ist, falls nicht binnen zweier Jahre Deutsch auf A2-Niveau des Gemeinsamen Europäischen Referenzrahmens für Sprachen nachgewiesen werden kann. Um aber einen dauerhaften Aufenthaltstitel mit uneingeschränktem Zugang zum Arbeitsmarkt zu erwerben, sind Deutschkenntnisse auf der Stufe B1 erforderlich. ${ }^{131}$ Das Ziel sei die „Integration rechtmäßig im Bundesgebiet niedergelassener Fremder"132 - und dazu zählen Asylwerber_innen, die sich im

126 Statistik Austria, Positiv rechtskräftige Asylentscheidungen 2000-2016 nach Staatsangehörigkeit, 23.8.2017, [http://www.statistik.at/wcm/idc/idcplg?ldcService=GET_PDF_FILE\&RevisionSelectionMethod=LatestReleased \&dDocName=109307], eingesehen 23.8.2017

127 Bundeskanzleramt Österreich, Rechtsinformationssystem, Bundesrecht konsolidiert: Gesamte Rechtsvorschrift für Herkunftsstaaten-Verordnung, Fassung vom 23.8.2017, 2017, [https://www.ris.bka.gv.at/GeltendeFassung.wxe ?Abfrage=Bundesnormen\&Gesetzesnummer=20006306\&ShowPrintPreview=False], eingesehen 23.8.2017.

128 Bundeskanzleramt Österreich, Rechtsinformationssystem, Spruch W212 1437203-1/3E, 27.3.2014, [https:// www.ris.bka.gv.at/Dokumente/Bvwg/BVWGT_20140327_W212_1437203_1_00/BVWGT_20140327_ W212_1437203_1_00.html], eingesehen 11.8.2017.

129 Maria Sterkl, Asylverfahren ohne Ende: Nach 18 Jahren gab Bangladescher auf, in: Der Standard online (2014), [http://derstandard.at/2000007974665/Asylverfahren-ohne-Ende-Nach-18-Jahren-gab-Bangladescher-auf], eingesehen 30.8.2017.

130 Heinz Fassmann, Migrations- und Integrationspolitik, in: Robert Kriechbaumer/Franz Schausberger (Hrsg.), Die umstrittene Wende. Österreich 2000-2006 (Schriftenreihe des Forschunsinstitutes für politisch-historische Studien der Dr.-Wilfried-Haslauer-Bibliothek, Salzburg 43), Wien-Köln-Weimar 2013, S. 695-712, hier S. 707.

131 Bundesministerium für Inneres Österreich, Niederlassung und Aufenthalt, o. D., [http://www.bmi.gv.at/cms/BMI_ Niederlassung/start.aspx\#t_Integrationsvereinbarung], eingesehen 11.8.2017.

132 Bundesministerium für Inneres Österreich, Niederlassung und Aufenthalt, eingesehen 11.8.2017. 
Verfahren befinden - um sie „zur Teilhabe am gesellschaftlichen, wirtschaftlichen und kulturellen Leben in Österreich [zu] befähigen"133.

Oftmals ist die finanzielle Situation eine Voraussetzung, um überhaupt am wirtschaftlichen und kulturellen Leben teilhaben zu können. Für Asylwerber_innen ist das jedoch schwierig, zumal sie nur in einem eingeschränkten Maß einer Arbeit nachkommen dürfen. Grundsätzlich steht ihnen der Arbeitsmarkt nach europäischem Recht zwar offen, jedoch können alle EU-Mitgliedsländer selber entscheiden, inwiefern und inwieweit sie das zulassen. ${ }^{134}$ De facto sind Asylwerber_innen in Österreich durch vielfache Bestimmungen und Voraussetzungen von der Ausübung unselbstständiger Tätigkeiten weitgehend ausgeschlossen, sofern es sich nicht um temporäre Saison- oder Erntehelferarbeiten handelt. ${ }^{135}$ Auch andere kleinere Beschäftigungen sind ihnen erlaubt, $z$. B. Tätigkeiten in den Flüchtlingsheimen, wo sie untergebracht sind oder gemeinnützige Arbeiten wie Landschaftspflege oder die Betreuung von Parkanlagen. ${ }^{136}$ Drei Monate nach Zulassung zum Asylverfahren ist die Aufnahme von selbstständigen Tätigkeiten möglich. ${ }^{137}$ Diese dürfen jedoch nicht der Gewerbeordnung unterliegen. ${ }^{138}$ Darunter fallen etwa Prostitution, Holzhacken, Rasenmähen oder der Kleinverkauf periodischer Druckwerke. Letzteres ist in der Praxis hauptsächlich der Verkauf von Straßenzeitungen. Weil alle Interviewpartner dieser Tätigkeit nachgehen und das für sie und viele andere Migrant_innen momentan ein zentraler Lebensinhalt ist, wird nachfolgend kurz auf die Tiroler Straßenzeitung 20er eingegangen.

\section{Der Verkauf von Straßenzeitungen in Tirol}

\subsection{Der "20er"}

Die Straßenzeitung 20er wurde 1996 durch den „Verein zur Förderung einer Straßenzeitung in Tirol“ konzipiert und im Dezember 1998 erstmals verkauft. ${ }^{139}$ Der Grundgedanke dahinter war, Menschen in sozialen Notlagen zu helfen, ihnen eine Perspektive zu geben und ein selbst erarbeitetes Einkommen möglich zu machen. ${ }^{140}$ Im Editorial der ersten Ausgabe heißt es: „Der zwanzger ist ein Projekt, das Menschen in sozialen Schwierigkeiten die Gelegenheit gibt, ihre Sprachlosigkeit zu überwinden und mit anderen in Kontakt zu treten."141 Wie bei allen Straßenzeitungen kaufen die Kolporteur_in-

\footnotetext{
133 Bundesministerium für Inneres Österreich, Niederlassung und Aufenthalt, eingesehen 11.8.2017.

134 Staller, Erwerbsarbeit als Integrationsinstanz, S. 28.

135 Ebd., S. 18-27.

136 Bundeskanzleramt Österreich, Rechtsinformationssystem, Bundesrecht konsolidiert: Gesamte Rechtsvorschrift für Grundversorgungsgesetz - Bund 2005, Fassung vom 30.8.2017, [https://www.ris.bka.gv.at/GeltendeFassung. wxe?Abfrage=Bundesnormen\&Gesetzesnummer=10005762\&ShowPrintPreview=False], eingesehen 30.8.2017.

137 Bundeskanzleramt Österreich, Rechtsinformationssystem, Bundesrecht konsolidiert: Gesamte Rechtsvorschrift für Grundversorgungsgesetz, eingesehen 30.8.2017.

138 Land Oberösterreich (Hrsg.), Dürfen Flüchtlinge in Österreich arbeiten?, o. D., S. 1, [http://www.landoberoesterreich.gv.at/Mediendateien/Formulare/DokumenteBH_GM/Beschaeftigung_Asylwerberlnnen.pdf\#sea $r c h=\% 22$ asylwerber\&\#×2b;besch\&\#x25;C3\&\#x25;A4ftigung\%22], eingesehen 12.8.2017. Staller, Erwerbsarbeit als Integrationsinstanz, S. 66.

140 Ebd.

141 20er-Die Tiroler Straßenzeitung, Nr. 1, Dezember 1998.
} 
nen die Exemplare um einen bestimmten Betrag und verkaufen sie dann auf der Straße um mindestens den doppelten Preis. ${ }^{142}$ Der 20er wird seit der Euro-Umstellung von den Verkäufer_innen um einen Euro gekauft und um zwei Euro verkauft.

In inhaltlicher Hinsicht widmeten sich die monatlichen Ausgaben anfangs immer speziellen Themen. Das Selbstverständnis der Zeitung war, als Sprachrohr für jene da zu sein, die den $20 e r$ auch verkauften. Das änderte sich nach schwächelnden Verkaufszahlen im Jahr 2004, als die Zeitung mit Erfolg neu konzipiert wurde. Anstatt den Themenheften wurde die inhaltliche Palette nun weiter gefasst. „Der 20er versteht sich seit da nicht mehr in einer Sprachrohrfunktion sondern [...] als Zeitung wie jede andere mit einem besonderen Vertriebsweg"143, erklärt Sylvia Riedmann-Flatz, die seither die Geschäftsführerin des 20ers ist.

Nach diesem Relaunch hat sich die Struktur des Verkäufer_innenpools allmählich aber doch gewandelt: Verkauften früher zum Großteil sozial Bedürftige mit österreichischer Staatsbürgerschaft die Zeitung, sind es seit der Einführung der bedarfsorientieren Mindestsicherung zu einem großen Teil Asylwerber_innen oder auch jene, die bereits einen positiven Asylbescheid und dennoch keine Arbeit haben. Riedmann-Flatz ortet die Einführung der bedarfsorientierten Mindestsicherung 2008 als einen ausschlaggebenden Faktor für diese Entwicklung. Bereits im Vorfeld - als sich Bund und Länder über die Regelung einig waren - sei zu erkennen gewesen, dass diejenigen, die anspruchsberechtigt waren, „langsam wegbrechen, aufhören, nicht müssen, nicht mehr mögen"144. Das Prinzip, sozial Bedürftigen zu helfen, sei jedoch immer dasselbe gewesen, egal, woher diese Personen stammen: „Ob das jetzt Einheimische waren oder nicht [...], das hat damals wie heute keine Rolle gespielt."145 Dass sich die Funktion des 20ers um eine Integrationsfunktion speziell für Migrant_innen erweitert hat, verneint Riedmann-Flatz, da eben auch Einheimische in sozialen Notlagen oftmals in irgendeiner Form wieder in die Gesellschaft integriert werden müssen.

Der Verkauf des 20ers stellt die Verkäufer_innen in die Öffentlichkeit und kann ihnen daher neben dem finanziellen Aspekt beim Erlernen und Verbessern der Sprache sowie dem Knüpfen von Kontakten helfen. Außerdem werden dabei viele andere grundlegende kulturelle Umgangsformen vermittelt, wie etwa das Einhalten „körperliche[n] Abstand[s], wenn man miteinander spricht, [die] Lautstärke des miteinander Sprechens, Stimmmodulationen, höfliche[n] Umgang, [oder] dass man sich an Regeln halten muss." ${ }^{146}$ Dafür werden die Verkäufer_innen zu Beginn der Tätigkeit in einem persönlichen Gespräch eingeschult. Letztlich ist der Verkauf von Straßenzeitungen für Asylwerber_innen eine der wenigen Tätigkeiten, denen sie legal nachkommen dürfen. Sie können dadurch dem zermürbenden Nichtstun entkommen, das gravierende Folgen für die psychische Gesundheit haben kann. ${ }^{147}$ Außerdem „engt Erwerbslosigkeit

142 Staller, Erwerbsarbeit als Integrationsinstanz, S. 60.

143 Tobias Leo, Interview mit Sylvia Riedmann-Flatz, Innsbruck, 5.7.2017, Aufnahme beim Verfasser, Anhang F, S. 100.

144 Leo, Interview mit Riedmann-Flatz, S. 100.

145 Ebd., S. 99

146 Ebd., S. 103

147 Staller, Erwerbsarbeit als Integrationsinstanz, S. 52. 
das Handlungsfeld ein [...] [und] die Spannungen zwischen Wollen und Können nehmen $z^{\prime \prime \prime}{ }^{\prime 14}$. Mit einem höheren Selbstbewusstsein sowie größeren finanziellen Möglichkeiten fällt es auf jeden Fall leichter, sich in eine (fremde) Gesellschaft zu integrieren und am sozialen und kulturellen Leben teilzunehmen.

\subsection{Zeitungsverkauf als Dritter Raum in einer postmigrantischen Gesellschaft}

Die relativ neue Denkrichtung einer postmigrantischen Gesellschaft stellt die Erfahrungen einer Gesellschaft mit Migration in den Mittelpunkt. Postmigrantisch bedeutet jedoch keine abgeschlossene Migration, sondern im Gegenteil, es wird akzeptiert, dass Migration stattgefunden hat, stattfindet und womöglich auch in Zukunft stattfinden wird. Das heißt, dass sich eine Gesellschaft mit ihren kulturellen, politischen und gesellschaftlichen Transformationen unter dem Begriff Migration sowohl im negativen als auch im positiven Sinne (immer wieder neu) konstituiert bzw. definiert. ${ }^{149}$ Daher kann auch gesagt werden: „Migration hat sich zu einem gesellschaftsstrukturierenden Metanarrativ entwickelt, das vielfach als allgemein erklärende Kategorie herangezogen wird."150 Die postmigrantische Perspektive hat das Ziel, die Bruchlinien innerhalb einer Gesellschaft sichtbar zu machen und will "Herkunft als kategoriale Trennlinie [...] überwinden [...] [sowie] hegemoniale Markierungen, Strukturen sowie Prozesse [entlarven] ${ }^{\prime 151}$.

Obwohl postmigrantische Theorien die politische Anerkennung als Einwanderungsland ${ }^{152}$ als Grundbedingung voraussetzt, ist das im Fall von Österreich nicht eindeutig. Dazu gibt es in der österreichischen politischen Landschaft widersprüchliche Auffassungen, die sich mitunter auch wandeln können. Faktisch ist es das aber, denn das letzte Jahr, in dem es eine negative Nettozuwanderung gab, war 1983. ${ }^{153}$ Auch innerhalb der Gesellschaft rückte das Thema Migration in jüngster Vergangenheit zunehmend in den Mittelpunkt. Harmonisch ist eine postmigrantische Gesellschaft keineswegs, denn über und mit dem Begriff der Migration werden Allianzen geschmiedet, Positionen ausverhandelt und Antagonismen durch Polarisierung verstärkt und somit deutlich sichtbar. ${ }^{154}$ Dadurch entsteht eine Dynamik, die „zu fundamentalen Veränderungen in über Jahrzehnte etablierten politischen, gesellschaftlichen und kulturellen Praktiken [führt], die von der Bevölkerung kontrovers diskutiert werden"155.

In diesem aus postmigrantischer Sicht dynamischen und ambivalenten Diskurs sind Zuwander_innen unfreiwillig der Hauptgegenstand der gesellschaftlichen Verhandlungen und damit in gewisser Weise abhängig davon. Besonders im politischen und

\footnotetext{
148 Staller, Erwerbsarbeit als Integrationsinstanz, S. 52.

149 Foroutan, Postmigrantische Gesellschaften, S. 233-234.

150 Ebd., S. 234.

151 Ebd., S. 249

152 Ebd., S. 239

153 Statistik Austria, Wanderungen mit dem Ausland (Außenwanderungen) nach In- und Ausländern 1961 bis 2016 , 23.8.2017, [http://www.statistik.at/wcm/idc/idcplg?ldcService=GET_PDF_FILE\&RevisionSelectionMethod=Lates tReleased\&dDocName=022920], eingesehen 26.8.2017.

154 Foroutan, Postmigrantische Gesellschaften, S. 239-247.

155 Ebd., S. 240.
} 
medialen Spektrum ist Migration praktisch untrennbar mit dem Begriff der Integration verbunden, vor allem, wenn es sich um Migration aus außereuropäischen Regionen handelt. Integration ist sicherlich kein Selbstläufer. Sie erfordert eine Gesellschaft, die auch bereit ist, Menschen mit einem anderen kulturellen Hintergrund aufzunehmen und auf der anderen Seite eine aktive Beteiligung und Bereitschaft der Migrant_innen, in die Gesellschaft einzutreten. Dabei muss die eigene Kultur nicht aufgegeben werden und ein Kulturtransfer muss nicht zwingend nur in eine Richtung gehen, wie es von manchen unter dem repressiven Begriff der Leitkultur eingefordert wird.

Es benötigt Räume, die beidseitig funktionieren, in denen die mitgebrachte Kultur gelebt werden kann und in denen genügend Platz und Möglichkeiten sichergestellt sind, sich in eine Gesellschaft positiv einfügen zu können. Im Bereich des Arbeitsmarktes ist, wie bereits erwähnt, der Platz in Österreich eher gering. Aber es existieren in diesem Bereich auch Nischen und eine davon ist der Verkauf von Straßenzeitungen. Auch Riedmann-Flatz spricht davon, dass der 20er ihren Verkäufer_innen einen „Raum [...] [mit] bestimmten Regeln"156 bietet. Die Präsenz in der Öffentlichkeit sowie die Verkaufssituationen selbst können in Anlehnung an Homi Bhabha auch als Dritte Räume begriffen werden, in denen kultureller Austausch stattfindet:

"It is that Third Space [...] which constitutes the discursive conditions of enunciation that ensure that the meaning and symbols of culture have no primordial unity or fixity; that even the same signs can be appropriated, translated, rehistoricized and read anew."157

Mit jedem Zeitungsverkauf, mit jedem Gespräch eröffnet sich ein Raum zur sozialen, kulturellen oder politischen Kommunikation. Im Idealfall werden von beiden Seiten vormals klar festgelegte Kategorien oder Annahmen widerlegt oder zumindest aus ihren vermeintlich fixen Verankerungen gelöst. In diesem Sinn kann eine beiderseitige Integration die Räume finden, um die Dynamik der Diskurse etwas zu entschärfen und zu entpolarisieren. Des Weiteren sieht Bhabha moderne Gesellschaften als hybride. Sein Begriff von Hybridität zielt jedoch nicht auf den Pluralismus innerhalb einer Gesellschaft ab. Für ihn ist „die gesamte Kultur [...] rund um Verhandlungen und Konflikte konstruiert" ${ }^{\prime 158}$. Dabei gehe es um (kulturelle) Autorität, wobei es aus der "Position des Underdogs [...] Möglichkeiten [gibt], die auferlegten kulturellen Autoritäten umzudrehen, einiges davon anzunehmen, anderes abzulehnen“159. Hybridität bedeutet demnach nicht einfach eine Vermischung "sondern strategische und selektive Aneignung von Bedeutungen, Raum schaffen für Handelnde, deren Freiheit und Gleichheit gefährdet sind"160.

Verkäufer_innen von Straßenzeitungen können daher durchaus die Rolle von Bhabhas Underdog einnehmen. Indem sie z. B. Geld verdienen oder Kontakte knüpfen, eröffnen

156 Leo, Interview mit Riedmann-Flatz, S. 104

157 Bhabha, The Location of Culture, S. 37.

158 Lukas Wieselberg, Interview mit Homi Bhabha, 9.11.2007, in: science.orf.at (Hrsg), 9.11.2007, [http://sciencev1.orf. at/news/149988.html], eingesehen 24.8.2017.

159 Wieselberg, Interview mit Homi Bhabha, eingesehen 24.8.2017.

160 Ebd. 
sich weitere Räume, etwa der Zugang zu kulturellen Veranstaltungen. Umgekehrt ist es dann innerhalb dieser Dritten Räume möglich, dass sich Autoritätssymbole wie Ressentiments oder Stereotype gegenüber Zuwanderern in ihrer Bedeutung oder Relevanz verändern. Diese sowohl konsens- als auch konfliktorientierte Ambivalenz in einer ebenso ambivalenten postmigrantischen Gesellschaft war bei den Interviewpartnern immer wieder zu finden. Einerseits bekamen sie gewisse Möglichkeiten zur Verfügung gestellt, für andere mussten sie kämpfen, wieder andere blieben vorerst verschlossen.

\subsection{Europa, Österreich und das "20er"-Verkaufen aus Sicht der Interviewten}

In den Gesprächen war deutlich zu erkennen, dass alle Interviewpartner ihren zukünftigen Lebensmittelpunkt in Österreich sehen. Manche Erwartungen an Europa haben sich erfüllt, einige auch nicht. Das Bild von Europa erscheint in Nigeria durch Mundpropaganda sowie Film und Fernsehen positiv überzeichnet, jedenfalls hat es ein attraktives Image und ist daher sicherlich auch anziehend: „It's a place where the light is constant, they have good road, they have good security [...] and the economy is good."161 Das korrupte System in Nigeria wird des Öfteren als negativer Vergleich genannt. Die Wahrnehmung der europäischen und österreichischen Politik ist dagegen überwiegend positiv: „The government love the people, although they have their own selfish agenda."162 Ebenso gibt es in Nigeria negative Ressentiments gegenüber Europäer_innen, wie etwa Bob erzählt: "My mindset was, [...] they were not friendly, they're authoritative and [...] they think us as a lesser race." ${ }^{163}$ Nach einiger Erfahrung revidierte er diese Ansicht: I got to discover, they are humans like me, they have feelings and they have emotions." ${ }^{\prime 164}$

Dass in Nigeria Europa von vielen Menschen als Paradies gesehen wird, bestätigt Chuis. Dieser aber "würde ihnen sagen, das ist nicht das Paradies, weil sieben Jahre warten ist auch nicht so einfach [...] [,] vor allem wenn du nichts tust, nur sitzen zuhause [sic!], essen"165. Die Deutschkurse und den Hauptschulabschluss habe er selber finanziert. Um das zu schaffen, hat er „fast drei Jahre [...] fast nur Reis und Tomatensauce [...] [oder] Brot [...] [von] Montag bis Sonntag"166 gegessen. Wenn das Leben nicht in Gefahr ist, würde er daher niemandem raten, nach Europa zu flüchten, denn dafür sei das Risiko zu groß. Insgesamt bleibt festzuhalten, dass alle Interviewpartner in einem friedlichen, liberalen und rechtsstaatlich geprägten Europa leben wollen und dafür bereit sind, viel auf sich zu nehmen. Dass sie mit einem niedrigen Lebensstandard zurechtkommen, liegt wohl auch an den Erfahrungen, die sie bisher in ihrem Leben gemacht haben.

An die meisten Gegebenheiten wie Kleidungsstil, Klima oder Kulinarik haben sich alle Interviewten schnell angepasst. Vor allem schätzen sie die Sicherheit, die Organisation und den Umgang miteinander in der Gesellschaft, im Vergleich zu Nigeria ist das kei-

161 Leo, Interview mit Eric, S. 93

162 Leo, Interview mit Bob, S.64.

163 Ebd., S. 62

164 Ebd.

165 Tobias Leo, Interview mit Chuis, Innsbruck, 12.7.2017, Aufnahme beim Verfasser, Anhang C, S. 76.

166 Leo, Interview mit Chuis, S. 76. 
ne Selbstverständlichkeit. Das Erlernen der deutschen Sprache betrachteten hingegen alle als das mit Abstand schwierigste Unterfangen. Das haben ihnen die zahlreichen Gespräche beim Verkaufen des 20ers erheblich erleichtert. In Bhabhas Sinn von den Dritten Räumen haben sie sich damit die Sprache - sofern sie als Autoritätssymbol betrachtet wird - angeeignet und damit neue Räume und Möglichkeiten erschlossen.

Abgesehen von der Sprache betont jeder der Befragten, wie wichtig das Knüpfen von Kontakten beim Verkauf gewesen sei. Die Öffentlichkeit ist sozusagen direkt konfrontiert mit den 20er-Verkäufer_innen. Zwar waren auch negative Erfahrungen dabei, jedoch überwiegt das Positive eindeutig bzw. sie stellen das Positive deutlich in den Vordergrund. Mit der Zeit - wohl auch, weil sie in der Regel längerfristig an einem Standort in der Öffentlichkeit stehen - bildeten sich so manche Freundschaften sowie gute Bekanntschaften. Diese so entstehenden persönlichen Netzwerke mit Menschen, die schon länger hier leben, sind mindestens so wichtig wie das Erlernen der Sprache und kommen dem Begriff der Integration im Prinzip näher. In dieser Hinsicht wurde wieder der zur Verfügung gestellte Raum genutzt und erweitert.

Auch der finanzielle Aspekt spielt eine tragende Rolle: Die Interviewpartner sind durch einen größeren Spielraum unabhängiger und zum Beispiel nicht mehr auf Kleiderspenden angewiesen. Alltagsgegenstände wie einen Laptop können sie sich wesentlich einfacher finanzieren. Möglich sei auch die Unterstützung der Angehörigen in Nigeria, wie Bob erklärt: „I earned five times much better than the money I was earning in my country [as an architect], so I could support my sister at that time in school." ${ }^{67}$ Außerdem besteht die Möglichkeit, wie etwa bei David, noch weiter in die Mitte der Gesellschaft zu rücken, indem statt in der Flüchtlingsunterkunft in einer Privatwohnung bzw. einer Wohngemeinschaft gelebt wird. Zusammen mit den Erlösen aus dem Zeitungsverkauf ist das möglich: Wenn Asylwerber_innen in privaten Unterkünften leben, werden sie gemäß dem Grundversorgungsgesetz mit insgesamt 365 Euro pro Monat für Verpflegung und Miete unterstützt. ${ }^{168}$

Psychologisch gesehen ist eine Beschäftigung besonders in der unsicheren Situation, in der sie sich als Asylwerber_innen jahrelang befinden, viel wert. „Es hilft uns, den Asylanten, [...] Stress [zu] vergessen"169, meint David. Auch eine Tages- und Wochenstruktur hat einen psychologischen Wert: "The newspaper is a lifesaver. [...] It helps you to have a reason for tomorrow [...]. And [it] gives me hope, I don't feel bored."170 Einige betonen auch, dass sie diese Tätigkeit als sehr wichtig einschätzen, um nicht in die Kriminalität abzurutschen. Unterm Strich bietet der Straßenzeitungsverkauf Asylwerber_innen einen Raum zur Integration, zur Teilhabe an der Gesellschaft sowie einen Raum für Hoffnung in unsicheren, prekären Lebensrealitäten.

167 Leo, Interview mit Bob, S. 61.

168 Günther Oswald, Wie viel Flüchtlinge in der Grundversorgung bekommen, in: Der Standard online (2017), [https://derstandard.at/2000068157547/Wie-viel-Fluechtlinge-in-der-Grundversorgung-bekommen], eingesehen 23.3.2018.

169 Leo, Interview mit David, S. 82.

170 Leo, Interview mit Bob, S. 66. 


\section{Fazit}

Es hat sich bestätigt, dass die gegenwärtigen und weitreichenden Probleme Nigerias tief historisch verwurzelt sind und sich gegenseitig bedingen. Auf die bereits in vorkoIonialer Zeit bestehenden religiösen Unterschiede - die nicht das Hauptproblem sind, aber bis heute immer wieder zu Spannungen führten sowie Konflikte religiös aufluden - folgten Zerwürfnisse in Wirtschaft, Politik, Kultur und Bildung. Im kollektiven Bewusstsein bzw. in den kollektiven „Bewusstseinen“ sind nur wenige positive Momente greifbar. Das ist auch in den Interviews deutlich hervorgegangen. Die vielen negativen Entwicklungen, welche sich in der dortigen Region in den letzten zweihundert Jahren vollzogen haben, scheinen in den Köpfen relativ präsent. Außerdem haben sich zahlreiche in der Kolonialzeit geschaffene Strukturen bis heute erhalten. Hohe Frustration, große Ungerechtigkeiten, ein niedriger Lebensstandard sowie fehlende Zukunftsperspektiven lassen für viele der Betroffenen den Glauben an eine gute Zukunft schwinden. Entsprechend der vielfältigen Probleme gibt es mindestens genauso viele Gründe, sich woanders eine Zukunft zu errichten oder zumindest zwischenzeitlich im Ausland eine Grundlage für ein besseres Leben aufzubauen. Die häufig zitierte Hilfe vor Ort würde im Fall von Nigeria, angesichts dieser in zwei Jahrhunderten gewachsenen hochkomplexen Problemlagen samt der überbordenden Korruption, wohl nicht viel ändern können.

Ebenfalls hat sich erwiesen, dass für eine Integration in eine Gesellschaft Zeit, Raum bzw. in beide Richtungen offene Dritte Räume sowie legale Beschäftigungsmöglichkeiten nötigt sind. Der 20er ist dafür ein gutes Beispiel innerhalb der aktuellen Gesetzeslage. In den Interviews entstand deutlich der Eindruck, dass die Gesprächspartner die Forderung nach Integration gerne erfüllen. Sie wollen hier bleiben, sich hier ein Leben aufbauen und sind froh, nicht mehr direkt mit den Problemen der nigerianischen Gesellschaft(en) konfrontiert zu sein. Im Gegenteil, sie wollen ein Leben in Sicherheit, ein Leben ohne Gewalt und in Wohlstand. Alle Interviewten wollten einer gewissen Ohnmacht entkommen und ihr Leben selber in die Hand nehmen. Sie sind bereit, sich aktiv in die Gesellschaft einzubringen, obwohl ihnen bewusst ist, dass sie nur eine geringe Chance haben, hier bleiben zu dürfen. Diese Chance wollen sie nützen, auch wenn ihr Leben mitten in der postmigrantischen Wohlstandsgesellschaft entbehrungsreich ist. Die Hoffnung ist groß und sie schätzen, dass sie überhaupt hier sind und diese Perspektive besteht. Das Aufnehmen einer legalen Tätigkeit wie der Verkauf von Straßenzeitungen eröffnet ihnen Möglichkeiten zur Integration. Die eingangs erwähnten Sprach- und Wertekurse greifen definitiv zu kurz, um die in der Integrationsvereinbarung definierten Ziele zur Teilhabe am wirtschaftlichen, kulturellen und gesellschaftlichen Leben zu erfüllen. Im Vergleich dazu „erfüllt" der Verkauf von Straßenzeitungen mittel- und langfristig diese Ansprüche viel deutlicher: Die Menschen haben ein Leben und existieren nicht nur. Sie lernen und festigen die Sprache, knüpfen Kontakte und haben die Möglichkeit, sich von diesen Dritten Räumen ausgehend weitere Räume in der Gesellschaft zu erschließen. 


\section{Literatur}

20er - Die Tiroler Straßenzeitung, Nr. 1, Dezember 1998.

Amassoma, Ditimi/Nwosa, Philip Ifeakachukwu, The Impact of Unemployment Rate on Productivity Growth in Nigeria: An Error Correcting Modeling Approach, in: International Journal of Economics and Management Services 2 (2013), Heft 8, S. 1-13, [https:// www.omicsonline.com/open-access/the-impact-of-unemployment-rate-on-productivity-growth-in-nigeria-an-error-correction-modeling-approach-2162-6359-2-145. pdf], eingesehen 11.06.2017.

Awofeso, Olu/Odeyemi, Temitayo Isaac, The Impact of Political Leadership and Corruption on Nigeria's Development since Independence, in: Journal of Sustainable Development 7 (2014), Heft 5, S. 240-253, [http://dx.doi.org/10.5539/jsd.v7n5p240], eingesehen 25.6.2017.

Baumer, Andreas, Irreguläre Migration und staatliche Politik in Spanien und Europa, Wiesbaden 2017.

Bhabha, Homi, The Location of Culture, London-New York 1994.

Bundeskanzleramt Österreich, Rechtsinformationssystem, Bundesrecht konsolidiert: Gesamte Rechtsvorschrift für Grundversorgungsgesetz - Bund 2005, Fassung vom 30.8.2017, [https://www.ris.bka.gv.at/GeltendeFassung.wxe?Abfrage=Bundesnormen \&Gesetzesnummer=10005762\&ShowPrintPreview=False], eingesehen 30.8.2017.

Bundeskanzleramt Österreich, Rechtsinformationssystem, Bundesrecht konsolidiert: Gesamte Rechtsvorschrift für Herkunftsstaaten-Verordnung, Fassung vom 23.8.2017, 23.8.2017, [https://www.ris.bka.gv.at/GeltendeFassung.wxe?Abfrage=Bundesnormen \&Gesetzesnummer=20006306\&ShowPrintPreview=False], eingesehen 23.8.2017.

Bundeskanzleramt Österreich Rechtsinformationssystem, Spruch W212 14372031/3E, 27.3.2014, [https://www.ris.bka.gv.at/Dokumente/Bvwg/BVWGT_20140327_ W212_1437203_1_00/BVWGT_20140327_W212_1437203_1_00.html], eingesehen 11.8.2017.

Bundesministerium für Inneres Österreich, Niederlassung und Aufenthalt, o. D., [http:// www.bmi.gv.at/cms/BMI_Niederlassung/start.aspx\#t_Integrationsvereinbarung], eingesehen 11.8.2017.

Bundesministerium für Inneres Österreich, Statistiken Asylwesen, o. D., [http://www. bmi.gv.at/cms/bmi_asylwesen/statistik/start.aspx], eingesehen 22.7.2017.

Davis, Thomas J./Kalu-Nwiwu, Azubike, Education, Ethnicity and National Integration in the History of Nigeria: Continuing Problems of Africa's Colonial Legacy, in: The Journal of Negro History 86 (2001), Heft 1, S. 1-11, [www.jstor.org/stable/1350175], eingesehen 16.6.2017.

Fassmann, Heinz, Migrations- und Integrationspolitik, in: Robert Kriechbaumer/Franz Schausberger (Hrsg.), Die umstrittene Wende. Österreich 2000-2006 (Schriftenreihe 
des Forschungsinstitutes für politisch-historische Studien der Dr.-Wilfried-HaslauerBibliothek, Salzburg 43), Wien-Köln-Weimar 2013, S. 695-712, hier S. 707.

Foroutan, Naika, Postmigrantische Gesellschaften, in: Heinz Ulrich Brinkmann/Martina Sauer (Hrsg.), Einwanderungsgesellschaft Deutschland. Entwicklung und Stand der Integration, Wiesbaden 2016.

Heerten, Lasse/Moses, A. Dirk, The Nigeria-Biafra war: postcolonial conflict and the question of genocide, in: Journal of Genocide Research 16 (2014), Heft 2-3, S. 169-203, [http://dx.doi.org/10.1080/14623528.2014.936700], eingesehen 22.6.2017.

Ifeoma, Nworgu Sharon, Living sacrifice among the Igbo, in: The Guardian online (2015), [https://guardian.ng/sunday-magazine/c180-arts/living-sacrifice-among-the-igbo/], eingesehen 25.11.2017.

Isiugo-Abanihe, Uche C./IOM Nigeria, Migration in Nigeria. A Country Profile 2014, Genf 2016, [https://publications.iom.int/system/files/pdf/mp_nigeria.pdf], eingesehen 26.6.2017.

Kappel, Robert, Nigeria - Ressourcenreichtum und Wachstum ohne Entwicklung, in: Matthias Basedau/Robert Kappel (Hrsg.), Machtquelle Erdöl. Die Außen-, Innen- und Wirtschaftspolitik von Erdölstaaten (Entwicklungstheorie und Entwicklungspolitik 11), Baden-Baden 2011, S. 141-166.

Klingholz, Reiner, Sklaven des Wachstums. Die Geschichte einer Befreiung, Frankfurt am Main-New York 2014.

Klingholz, Reiner/Lill, Felix/Budde, Joachim, Wachsen und Schrumpfen. Nigeria: Außer Kontrolle, in: Zeit Online (2014), [http://www.zeit.de/2014/07/demografie-iran-nigeriajapan-deutschland/seite-2], eingesehen 29.8.2017.

Land Oberösterreich, Dürfen Flüchtlinge in Österreich arbeiten?, O. D., [http://www. land-oberoesterreich.gv.at/Mediendateien/Formulare/DokumenteBH_GM/Beschaeftigung_Asylwerberlnnen.pdf\#search=\%22asylwerber\&\#x2b;besch\&\#x25;C3\&\#x25;A4fti gung\%22], eingesehen 12.8.2017.

National Bureau of Statistics Nigeria, 2015 Statistical Report on Women and Men in Nigeria, o. O. (Abuja) 2016, [http://www.nigerianstat.gov.ng/download/491], eingesehen 10.6.2017.

O. A., 'Boko Haram attack' frees hundreds of prisoners, in: BBC online, 8.9.2010, [http:// www.bbc.com/news/world-africa-11229740], eingesehen 22.6.2017.

Odeyemi, Jacob Oluwole, A Political History of Nigeria and the Crisis of Ethnicity in Nation-Building, in: International Journal of Developing Societies 3 (2014), Heft 1, S. 87-95, [https://doi.org/10.11634/216817831504459], eingesehen 20.6.2017.

Ogbeidi, Michael M., Political Leadership and Corruption in Nigeria Since 1960: A Socioeconomic Analysis, in: Journal of Nigeria Studies 1 (2012), Heft 2, S. 1-25, [www.unh.edu/ nigerianstudies/articles/.../Political_leadership.pdf], eingesehen 25.6.2017. 
O'Neill, Tom, Curse of the Black Gold. Hope and betrayal on the Niger Delta, in: National Geographic online (2007), [http://ngm.nationalgeographic.com/2007/02/nigerian-oil/ oneill-text], eingesehen 12.6.2017.

Onyibor, Marcel Ikechukwu Sunday, National Identity and Crisis of Integration in MultiEthnic Nigeria: An Existentialist Perpective, in: Open Journal of Philosophy 6 (2016), S. 1-12, [http://www.dx.doi.org/10.4236/ojpp.2016.61001], eingesehen 20.6.2017.

Osaghae, Eghosa, Crippled Giant. Nigeria since Independence, London 1998.

Oswald, Günther, Wie viel Flüchtlinge in der Grundversorgung bekommen, in: Der Standard online (2017), [https://derstandard.at/2000068157547/Wie-viel-Fluechtlingein-der-Grundversorgung-bekommen], eingesehen 23.3.2018.

Ross, Will, Nigeria elections: Buhari scores historic win, in: BBC online, 1.4.2015, [http:// www.bbc.com/news/world-africa-32141703], eingesehen 22.6.2017.

Salomon, Stefan, The Tragedy of Nigerian Society, in: Andreas Exenberger (Hrsg.), Afrika - Kontinent der Extreme (Edition Weltordnung - Religion - Gewalt 9), Innsbruck 2001, S. 147-168, hier S. 147, [http://diglib.uibk.ac.at/urn:nbn:at:at-ubi:3-472], eingesehen 11.6.2017.

Staller, Julia, Erwerbsarbeit als Integrationsinstanz bei Asylwerberlnnen in Österreich am Beispiel der Tiroler Straßenzeitung 20er, Dipl. Innsbruck 2012.

Statistik Austria, Bevölkerung am 1.1.2017 nach detailliertem Geburtsland und Bundesland, 23.5.2017, [http://www.statistik.at/wcm/idc/idcplg?ldcService=GET_PDF_FILE\&R evisionSelectionMethod=LatestReleased\&dDocName=023841], eingesehen 7.7.2017.

Statistik Austria, Bevölkerung zu Jahresbeginn 2002-2017 nach detaillierter Staatsangehörigkeit, 23.5.2017, [http://www.statistik.at/wcm/idc/idcplg?ldcService=GET_ PDF_FILE\&RevisionSelectionMethod=LatestReleased\&dDocName=037044], eingesehen 7.7.2017.

Statistik Austria, Positiv rechtskräftige Asylentscheidungen 2000-2016 nach Staatsangehörigkeit, 23.8.2017, [http://www.statistik.at/wcm/idc/idcplg?ldcService=GET_ PDF_FILE\&RevisionSelectionMethod=LatestReleased\&dDocName=109307], eingesehen 23.8.2017.

Statistik Austria, Statistisches Jahrbuch 2017, Wien 2017, [https://www.statistik.at/wcm/ idc/idcplg?IdcService=GET_PDF_FILE\&dDocName=054401], eingesehen 7.7.2017.

Statistik Austria, Wanderungen mit dem Ausland (Außenwanderungen) nach Inund Ausländern 1961 bis 2016, 23.8.2017, [http://www.statistik.at/wcm/idc/idcplg? IdcService=GET_PDF_FILE\&RevisionSelectionMethod=LatestReleased\&dDocNa $\mathrm{me}=022920]$, eingesehen 26.8.2017.

Sterkl, Maria, Asylverfahren ohne Ende: Nach 18 Jahren gab Bangladescher auf, in: Der Standard online (2014), [http://derstandard.at/2000007974665/Asylverfahren-ohneEnde-Nach-18-Jahren-gab-Bangladescher-auf], eingesehen 30.8.2017. 
Transparency International, Corruption Perceptions Index 2016, 25.1.2016, [https:// www.transparency.org/news/feature/corruption_perceptions_index_2016], eingesehen 16.7.2017.

Wieselberg, Lukas, Interview mit Homi Bhabha, 9.11.2007, in: science.orf.at (Hrsg), 9.11.2007, [http://sciencev1.orf.at/news/149988.html], eingesehen 24.8.2017.

Tobias Leo ist Student des Masterstudiums Geschichte an der Universität Innsbruck. Tobias.Leo@student.uibk.ac.at

\section{Zitation dieses Beitrages}

Tobias Leo, „A Place where the Light is constant". Faktoren der Migration von Nigeria nach Europa und Integration durch den Verkauf von Straßenzeitungen am Beispiel von Tirol?, in: historia.scribere 10 (2018), S. 111-140, [http://historia.scribere.at], eingesehen 19.6.2018 (=aktuelles Datum).

(C) Creative Commons Licences 3.0 Österreich unter Wahrung der Urheberrechte der Autorlnnen. 